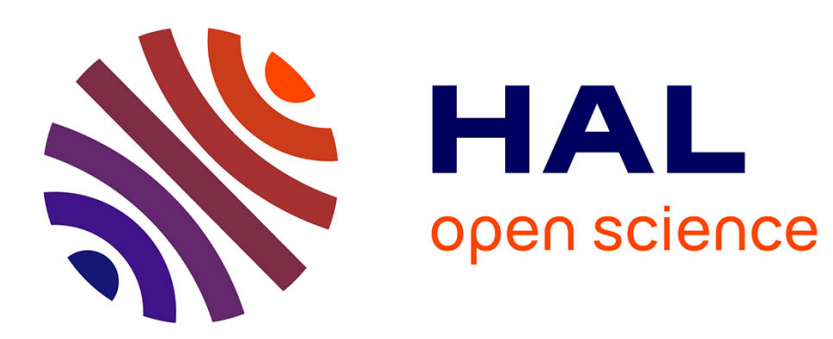

\title{
A unified model for adhesive interfaces with damage, viscosity and friction
}

Gianpietro del Piero, Michel Raous

\section{To cite this version:}

Gianpietro del Piero, Michel Raous. A unified model for adhesive interfaces with damage, viscosity and friction. European Journal of Mechanics - A/Solids, 2010, 29 (4), pp.496-507. hal-00462116

\section{HAL Id: hal-00462116 https://hal.science/hal-00462116}

Submitted on 24 Mar 2010

HAL is a multi-disciplinary open access archive for the deposit and dissemination of scientific research documents, whether they are published or not. The documents may come from teaching and research institutions in France or abroad, or from public or private research centers.
L'archive ouverte pluridisciplinaire HAL, est destinée au dépôt et à la diffusion de documents scientifiques de niveau recherche, publiés ou non, émanant des établissements d'enseignement et de recherche français ou étrangers, des laboratoires publics ou privés. 


\title{
A unified model for adhesive interfaces with damage, viscosity, and friction
}

\author{
Gianpietro Del Piero \\ Dipartimento di Ingegneria, Università di Ferrara, via Saragat 1, 44100 Ferrara, Italy \\ dlpgpt@unife.it \\ Michel Raous \\ Laboratoire de Mécanique et d'Acoustique, CNRS, 31 Chemin Joseph Aiguier 13402, Marseille cedex 20, France \\ raous@1ma.cnrs-mrs.fr \\ (Corresponding Author. Phone: 00334911640 53, Fax: 00334911644 81)
}

\begin{abstract}
A general framework for models describing adhesive contact between rigid bodies is proposed. The intensity of adhesion is supposed to decrease under the action of prescribed tangential and normal relative displacements. The reduction is attributed to progressive damage, and comes with energy dissipation. Additional dissipation due to viscosity and friction is also taken into account. The response of the interface is described by a single state variable. It is determined by general laws expressing a mechanical version of the first two laws of thermodynamics, combined with a set of phenomenological assumptions.
\end{abstract}

Key words: Adhesive contact, cohesive interface.

\section{1 - Introduction}

During the last few decades, several models for cohesive interfaces have been developed. Many papers deal with cohesive zone models in fracture mechanics (Dugdale, 1960; Barenblatt, 1962; Needleman, 1987, 1990, 1992; Tvergaard, 1990; Tvergaard and Hutchinson, 1992, 1996; Xu and Needleman, 1994; Costanzo and Walton, 1997; Needleman and Rosakis, 1999; Monerie, 2000; Péralès, 2005; Brinckmann and Siegmund, 2008). Other models are devoted to specific modes of interface decohesion in composite materials, such as delamination (Allix et al, 1995), peeling (Nguyen and Levy, 2009), or fiber debonding (Michel and Suquet, 1994; Raous et al, 1999; Monerie, 2000; Raous and Monerie, 2002). In Geophysics, the two main classes of interface models for studying fault nucleation, "rate and state" and "slip weakening" laws (Campillo and Ionescu, 1997; Rice and Ruina, 1983; Uenishi and Rice, 2003), can also be considered among the cohesive zone models. Finally, there is a number of models dealing in a more general way with adhesive interfaces (Frémond, 1987, 1988; Raous, 1999; Raous et al, 1999; Chaboche et al, 2001; Raous and Monerie, 2002; Talon and Curnier, 2003; Freddi and Frémond, 2006). This list is by no means exhaustive; other references can be found in the papers cited above.

In most theories, the loss of cohesion is described by a damage variable, called intensity of adhesion by Frémond $(1987,1988)$. This variable is defined on the interface, considered as a material boundary with a null thickness. Initially, adhesion was studied only under monotonic loading conditions, so that the problem of the reversibility of the material response was not taken into consideration. Only after the 1990's, with the study of cyclic behavior, it became standard to consider damage as irreversible. An exception to this generally accepted trend is the model of adhesion with healing recently proposed by Raous et al (2006), in which adhesion is supposed to recover, partially or totally, when contact is restored after total separation.

In the contributions cited above, different assumptions have been made regarding the main characteristics of the interface behavior:

- for normal relative displacements, the non-interpenetration condition has been enforced either by unilateral conditions, or by compliance or penalization devices,

- for tangential relative displacements, either frictional or frictionless contact has been considered; in frictional models, the transition between the initial regime of full adhesion and the final purely frictional regime has been assumed to be either progressive or brutal,

- some models consider rate dependent effects such as viscosity, while other ones are rate independent,

- the coupling between normal and tangential effects is often underestimated. This is mainly because in most applications one of them is dominating. 
The aim of the present paper is to provide a relatively general theoretical framework for adhesive contact. In our model the loss of adhesion is identified with irreversible damage, and the intensity of damage is assumed to depend on the coupled effect of normal and tangential deformation. Moreover, we assume unilateral conditions for normal displacements and Coulomb friction for tangential displacements. We also assume that the intensity of friction grows progressively with damage, and that the dissipation includes a part due to viscosity.

The proposed framework is based on the definition of a small number of fundamental objects:

- general laws, typically, energy conservation and dissipation principle, that is, mechanical versions of the first two laws of thermodynamics,

- $\quad$ a set of state variables, that is, an array of independent variables which fully determine the response to all possible deformation processes,

- a set of elastic potentials and dissipation potentials, which are functions of state in terms of which the general laws take specific forms,

- a set of constitutive assumptions.

The choice of the state variables, of the potentials, and of the constitutive equations is clearly influenced by the phenomenological features of the interface. It is at this level that specific microstructural schemes or failure mechanisms enter the play. We proceed to our construction systematically throughout Section 2. In Subsection 2.1 we start from the simplest case of a purely normal deformation with adhesion, in which adhesion is assumed to decrease progressively with increasing damage. Then we gradually enrich the initial scheme by introducing viscous dissipation in Subsection 2.2, and tangential deformation in Subsection 2.3. Finally, in Subsection 2.5 we discuss the case of coupled normal and tangential deformation in the presence of damage, viscosity, and friction.

The model is based on a single state variable which, together with the normal and tangential relative displacements at the interface, defines all elastic and dissipation potentials. The state variable measures the current intensity of damage, and is strictly related to the intensity of adhesion introduced by Frémond (1987, 1988). The choice of a single state variable reflects the idea that the intensity of damage is determined by the coupled effects of normal and tangential loading.

In the case of purely normal deformation, the basic experimental information that we assume to be known is the loading curve. This is the force-displacement relation in a monotonic deformation process starting from the virgin state. In the case of coupled normal and tangential deformation we show that, interestingly enough, the constitutive parameters are not determined by two separate loading curves, one for the normal and one for the tangential deformation, but by a single curve corresponding to an appropriate diagonal experiment.

Usually the power equation, which comes from time differentiation of the equation of energy conservation, is sufficient to determine the evolution of the state variable. However, there are situations in which the power equation is identically satisfied. Typically this is the case when dissipation due to damage is present, and viscous dissipation is neglected. In such situations, the evolution of the state variable is determined by the subsequent derivatives of the power equation. In the presence of physical constraints expressed by inequalities, for example, non-interpenetration, different responses at loading and unloading are obtained.

The efficiency of the theoretical scheme is tested on a specific process involving both normal and tangential deformations. This is done in Subsection 2.4 in the case of dissipation due to damage, and in Subsection 2.5 for dissipation due to damage, viscosity and friction. The response of the interface is determined qualitatively, without recourse to a numerical code, and the results are quite realistic. In the final Section 3 we show that, by an appropriate choice of the potentials and of the constitutive equations, the RCCM model developed in (Raous et al, 1999; Raous and Monerie, 2002), fits the proposed general scheme.

The RCCM model has been applied to more general situations, such as 2D and 3D deformable bodies instead of 2D rigid bodies (Raous et al, 1999; Raous and Monerie, 2002). Similar generalizations can be made for the model presented here. We do not discuss this possibility, since our effort is rather directed to the construction of a systematic model starting from a minimal number of general assumptions.

\section{Construction of a general model for adhesive interfaces}

Consider two rigid bodies separated by a plane material interface of negligible thickness. We take $u$ and $v$ to be the normal and tangential components, respectively, of the relative displacement of the surfaces of the two bodies. We also take $\sigma$ and $\tau$ to be the normal and tangential components of the force transmitted across the interface. The restriction

$$
u \geq 0
$$


is imposed to prevent interpenetration. We assume that a state of the interface is defined by the triplet $(u, v, \alpha)$, where $\alpha$ is a state variable measuring the current level of damage. Our purpose is to determine the evolution $t \mapsto \alpha_{(t)}$ of $\alpha$ in a deformation process $t \mapsto\left(u_{(t)}, v_{(t)}\right)$ starting from a given initial state $\alpha_{(0)}=\alpha_{0}$. The response curves $(\sigma, u)$ and $(\tau, v)$ then follow from the assumed constitutive equations.

We construct our model by steps, beginning from the case of purely normal loading, in which the tangential displacement and the tangential force are absent. We initially consider the simplest case in which the only source of dissipation is the damage due to the decrease in the adhesion intensity, and only later we will take into account the additional sources of dissipation provided by viscosity and friction.

\subsection{Adhesion with purely normal damage}

Consider an interface in which the damage is purely normal. In this case it is sufficient to prescribe a normal displacement $u$, determining a force $\sigma$ normal to the contact surface. A deformation process then reduces to the function $t \mapsto u_{(t)}$. We are interested in the following type of response:

(i) there is a function $f$ which determines the force $\sigma_{(t)}=f\left(u_{(t)}\right)$ at first loading, that is, in a monotonic nondecreasing process $t \mapsto u_{(t)}$ from the virgin state $\left(u_{(0)}, \alpha_{(0)}\right)=(0,0)$.

(ii) at unloading, $\sigma$ decreases linearly down to the origin,

(iii) during the subsequent reloading process the unloading line is crossed backwards, and when the loading curve $\sigma=f(u)$ is reached again, the reloading process continues along that curve,

(iv) there is a critical value $u_{r}$ of $u$ such that, after this value has been reached, the force takes permanently the value $\sigma=0$,

(v) when $u=0$, a compressive force $\sigma$ of arbitrary intensity can be transmitted across the surface.

We assume that the function $f$ is defined for all $u \geq 0$, positive in the open interval $\left(0, u_{r}\right)$ and null outside, and that it has the general shape shown in Fig. 1a. The laws assumed in the papers mentioned in the Introduction all have this general shape. Its restriction to $\left(0, u_{r}\right)$ is assumed to be star-shaped with respect to the origin. That is, every line from the origin has at most one intersection with this curve. Consequently, the function

$$
g(u):=\frac{f(u)}{u}
$$

is strictly decreasing for $u<u_{r}$ and is identically zero for $u \geq u_{r}$. The function $g$ measures the current stiffness of the interface, while the critical value $u_{r}$ corresponds to complete rupture of the interfacial bonds.
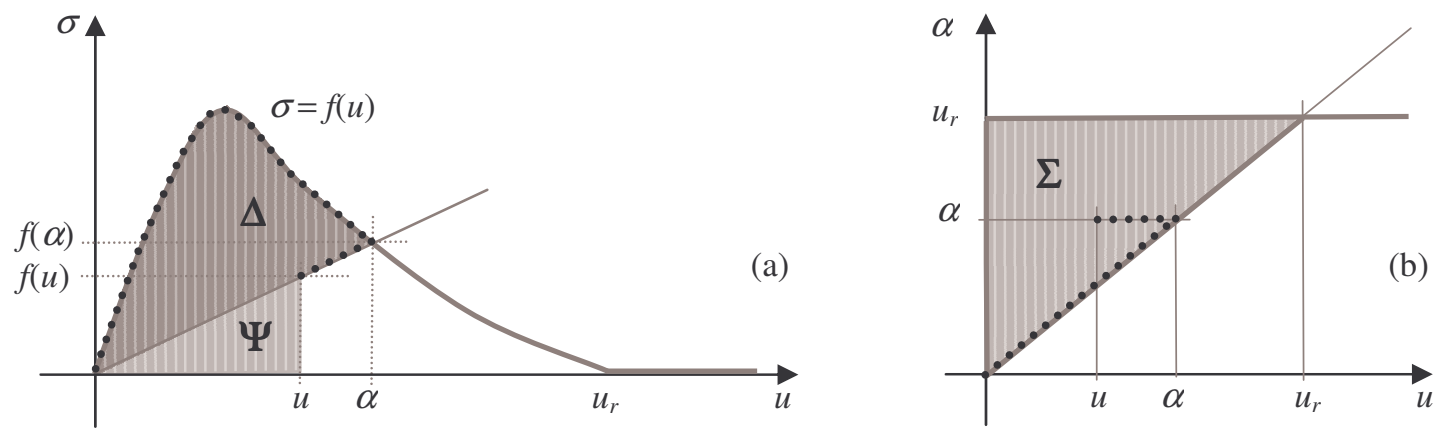

Figure 1. Adhesion with damage under normal loading. Response to a deformation process of loadingunloading starting from the origin, represented in the force-displacement plane (a), and in the state space (b).

There are only two types of pairs $(\sigma, u)$ which are admissible for this type of response, in the sense that they can be reached in a deformation process $t \mapsto u_{(t)}$ starting from the origin:

- those with $0 \leq u<u_{r}$ and $0 \leq \sigma \leq f(u)$,

- those with $u \geq u_{r}$ and $\sigma=0$.

Though the behavior of the interface, as defined by items (i)-(v) above, is fully described in terms of the variables $\sigma$ and $u$, in view of subsequent developments we find it convenient to introduce a state variable $\alpha$, defined over the admissible pairs $(\sigma, u)$ in the following way: 


$$
\begin{array}{ccc}
\alpha \in\left[0, u_{r}\right] & \text { if } & u=0, \\
g(\alpha)=\sigma / u & \text { if } & 0<u<u_{r}, \\
\alpha=u_{r} & \text { if } & u \geq u_{r} .
\end{array}
$$

Notice the indeterminacy of $\alpha$ for $u=0$, while, by the assumed monotonicity of $g$, the equation $g(\alpha)=\sigma / u$ defines $\alpha$ unambiguously for all $u$ in $\left(0, u_{r}\right)$. Conversely, every pair $(\sigma, \alpha)$ with

$$
\sigma<f(\alpha) \text { if } \alpha<u_{r}, \quad \sigma=0 \quad \text { if } \alpha \geq u_{r},
$$

defines one and only one pair $(\sigma, u)$.

According to the definition given for $\alpha$, the loading curve is made of points $(\sigma, u)$ with $u=\alpha$ and $\sigma=f(\alpha)$. For points $(\sigma, u)$ not on the loading curve, $\alpha$ is the abscissa of the intersection of the loading curve with the line joining the point $(\sigma, u)$ with the origin. The inequality

$$
\alpha \geq u
$$

holds at all points $(\sigma, u)$ with $u$ in $\left(0, u_{r}\right)$.

A pair $(u, \alpha)$ is a state of the interface. A state is admissible if the corresponding pair $(\sigma, u)$ is admissible. The set of all admissible states

$$
\Sigma=\left\{(u, \alpha) \mid u_{r} \geq \alpha \geq u \geq 0\right\} \cup\left\{(u, \alpha) \mid \alpha=u_{r}, u \geq u_{r}\right\},
$$

is the state space.

In Fig.1a, the response to first loading up to $\alpha$ followed by unloading up to $u$ is shown by a dotted line in the $(\sigma, u)$ plane. From the figure it is clear that, before complete rupture is achieved, $\alpha$ represents the largest displacement reached in a deformation process up to the current instant. The state space is represented in Fig.1b, where the dotted line shows the trajectory in the state space corresponding to the trajectory in the forcedisplacement plane $(\sigma, u)$. The dotted line follows the $\alpha=u$ line during the loading regime, and a horizontal line during the subsequent unloading regime.

In what follows the response described above in items (i)-(v) is described mathematically by specifying the evolution law $t \mapsto \alpha(t)$ for the state variable. To do this we use the elastic potential (6), the dissipation potential (8), the general laws (10), (11), and the constitutive equation (19) below. This approach may look formal and of little use. In fact, its importance relies on fixing a systematic procedure, to be applied later to far more general situations.

We start from the observation that, in the response described above, the area denoted by $\Psi$ in Fig. 1a represents recoverable work, since this area vanishes when unloading proceeds up to the origin. Moreover, the area denoted by $\Delta$ represents an irreversibly lost work, since no point in this area is accessible from the point $\left(u_{(t)}, \alpha_{(t)}\right)$. Accordingly, for any state $(u, \alpha)$ we take the area $\Psi$ to define the elastic strain energy

$$
\Psi(u, \alpha)=\frac{1}{2} g(\alpha) u^{2},
$$

and we take the area $\Delta$ to define the dissipated work

$$
\Delta(\alpha)=\int_{0}^{\alpha} f(s) d s-\frac{1}{2} g(\alpha) \alpha^{2} .
$$

This is the work spent in a loading-unloading cyclic process from $u=0$ to $u=\alpha$ and back. It can be deduced from the dissipation potential

$$
\Phi_{\mathrm{d}}(\alpha, \dot{\alpha})=-\frac{1}{2} g^{\prime}(\alpha) \alpha^{2} \dot{\alpha}
$$

Indeed, the dissipation power associated with $\Phi_{\mathrm{d}}$

$$
D_{\mathrm{d}}(\alpha, \dot{\alpha})=\dot{\alpha} \frac{\partial}{\partial \dot{\alpha}} \Phi_{\mathrm{d}}(\alpha, \dot{\alpha})=-\frac{1}{2} g^{\prime}(\alpha) \alpha^{2} \dot{\alpha}
$$

coincides with the time derivative $\dot{\Delta}(\alpha)$ of $\Delta(\alpha)$. We note that this coincidence is a characteristics of rate independent materials. Indeed, the response defined above is insensitive to time rescaling. Note also that the derivative of $\Phi_{\mathrm{d}}$ with respect to $\dot{\alpha}$ is the generalized force associated with the generalized velocity $\dot{\alpha}$, see e.g. (Halphen and Nguyen 1975).

In any process $t \mapsto u_{(t)}$, the work performed in a time interval $\left(t_{1}, t_{2}\right)$ is equal to the sum of the changes in the energy and the dissipation over the same interval:

$$
W_{\left(t_{1}, t_{2}\right)}=\Psi_{\left(t_{2}\right)}-\Psi_{\left(t_{1}\right)}+\Delta_{\left(t_{2}\right)}-\Delta_{\left(t_{1}\right)} .
$$

This equation expresses the conservation of energy, in the absence of applied loads and when inertia effects are negligible. This equation and the dissipation inequality

$$
D_{\mathrm{d}}(\alpha, \dot{\alpha}) \geq 0,
$$


which is the purely mechanical version of the Clausius-Duhem inequality, see e.g. (Truesdell \& Noll 1965, Sect. 79), are the mechanical counterparts of the first two laws of thermodynamics. The inequality is satisfied if and only if

$$
\dot{\alpha} \geq 0 \text {. }
$$

Indeed, if $\alpha>0$, the inequality $\dot{\alpha} \geq 0$ follows from the negativeness of $g^{\prime}(\alpha)$. If $\alpha=0$, the same inequality follows from the fact that $\alpha$ cannot decrease, because it cannot take negative values.

By time differentiation of (10) we obtain the power equation

$$
P_{(t)}=\dot{\Psi}_{(t)}+\dot{\Delta}_{(t)},
$$

which is therefore a necessary condition for energy conservation. In the present model, the external power is

the time derivative of $\Psi$ is

$$
P_{(t)}=\sigma_{(t)} \dot{u}_{(t)},
$$

$$
\dot{\Psi}(u, \alpha)=\frac{1}{2} g^{\prime}(\alpha) u^{2} \dot{\alpha}+g(\alpha) u \dot{u},
$$

and the time derivative of $\Delta$ is given by (9). With $\Psi, \Delta, P$ as in (6), (7), (14), the power equation becomes

$$
(\sigma-g(\alpha) u) \dot{u}+\frac{1}{2} g^{\prime}(\alpha)\left(\alpha^{2}-u^{2}\right) \dot{\alpha}=0 .
$$

Due to the restrictions on the sign of each factor, the term $g^{\prime}(\alpha)\left(\alpha^{2}-u^{2}\right) \dot{\alpha}$ is non-positive. Then, one has

$$
(\sigma-g(\alpha) u) \dot{u} \geq 0
$$

for any $\dot{u}$. In particular, if $u>0$, the sign of $\dot{u}$ is free, and one has

$$
\sigma=g(\alpha) u \geq 0 \quad \text { if } u>0 .
$$

In this way, the relation (3) between $\sigma, \alpha$, and $u$ when $0<u<u_{r}$ has been re-obtained. At $u=0$, one is free of choosing any constitutive relation compatible with inequality (17). In view of the requirement (v) about the unilateral contact, we introduce the decomposition $\sigma=\sigma^{+}-\sigma^{-}$, with

$$
\sigma^{+}=\max \{\sigma, 0\}, \quad \sigma^{-}=\max \{-\sigma, 0\},
$$

and for $\sigma^{+}$we take the constitutive equation

$$
\sigma^{+}=g(\alpha) u .
$$

For $\sigma^{-}$we observe that, when $\sigma<0$ and $u=0$, inequality (17) reduces to $-\sigma^{-} \dot{u} \geq 0$. Because $\dot{u} \geq 0$ by (1) and $\sigma^{-} \geq 0$ by its very definition, it must be $\sigma^{-} \dot{u}=0$. In this way, the Signorini unilateral contact law

$$
\sigma^{-} \geq 0, \quad u \geq 0, \quad \sigma^{-} u=0,
$$

is obtained. In particular, this law tells us that $\sigma^{-}=0$ for all $u>0$. When $u=0$ and $\dot{u}>0$, it follows from (17) that $\sigma \geq 0$, and therefore $\sigma^{-}=0$. This leads to the unilateral contact law for the velocities

$$
\sigma^{-} \geq 0, \quad \dot{u} \geq 0, \quad \sigma^{-} \dot{u}=0 \quad \text { if } u=0 .
$$

The two laws together tell us that $\sigma^{-}$is non-zero only if $u=\dot{u}=0$.

From equation (19) we have that $\sigma-g(\alpha) u=0$ when $\sigma \geq 0$, and from (21) we have that $\dot{u}=0$ when $\sigma<0$. Hence, the equality $(\sigma-g(\alpha) u) \dot{u}=0$ holds in all cases. The power equation (16) then reduces to

$$
g^{\prime}(\alpha)\left(\alpha^{2}-u^{2}\right) \dot{\alpha}=0 .
$$

To determine the evolution law for $\alpha$, we partition the state space into the four subsets listed below, and we determine separately the evolution of $\alpha$ in each subset.

(i) In the subset $\left\{0 \leq u<\alpha<u_{r}\right\}$, both $g^{\prime}(\alpha)$ and $\left(\alpha^{2}-u^{2}\right)$ are different from zero. Therefore, equation (22) gives $\dot{\alpha}=0$. The interface evolves according to the linear elastic law $\sigma=g(\alpha) u$, with constant stiffness $g(\alpha)$.

(ii) In the subset $\left\{0<u=\alpha<u_{r}\right\}$, equation (22) is satisfied identically. A necessary condition for energy conservation is then that the second time derivative of equation (10), that is, the first time derivative of (16), be zero:

$$
((\sigma-g(\alpha) u) \dot{u})^{\cdot}+\frac{1}{2}\left(g^{\prime}(\alpha) \dot{\alpha}\right)^{\cdot}\left(\alpha^{2}-u^{2}\right)+g^{\prime}(\alpha)(\alpha \dot{\alpha}-u \dot{u}) \dot{\alpha}=0,
$$

and since $\sigma=g(\alpha) u$ and $g^{\prime}(\alpha)<0$ at both the current and the following instants, this equation reduces to 


$$
(\dot{\alpha}-\dot{u}) \dot{\alpha}=0 .
$$

For $\dot{u} \leq 0$, condition $\dot{\alpha} \geq 0$ implies $\dot{\alpha}=0$. If $\dot{u}>0$, from the observation that $\alpha=u$ at the current instant and $\alpha \geq u$ at all instants implies $\dot{\alpha} \geq \dot{u}$, we deduce that $\dot{\alpha}=\dot{u}$.

(iii) At the point $\{0=u=\alpha\}$ equations (22) and (24) are satisfied identically, and the vanishing of the next time derivative of the energy is required. By differentiating equation (23) at $\alpha=u=0$, one obtains

$$
\left(\dot{\alpha}^{2}-\dot{u}^{2}\right) \dot{\alpha}=0
$$

and because $\alpha=u=0$ implies $\dot{\alpha} \geq \dot{u}$, it follows that $\dot{\alpha}=\dot{u}$.

(iv) Finally, in the subset $\left\{\alpha=u_{r}\right\} \dot{\alpha}$ is zero, because $\alpha$ cannot decrease because of the dissipation inequality $\dot{\alpha} \geq 0$, and cannot increase because it cannot take values greater than $u_{r}$.

The above conclusions can be collected in the formula

$$
\dot{\alpha}=\left\{\begin{array}{l}
\dot{u} \text { if } \alpha=u<u_{r} \text { and } \dot{u}>0, \\
0 \quad \text { otherwise, }
\end{array}\right.
$$

which specifies the evolution of $\alpha$ in all possible cases. This is an evolution law of the same type as the DruckerPrager law in plasticity. We have shown that this evolution law is determined by the assumptions $u \geq 0$ and $u \leq \alpha \leq u_{r}$, the general laws (10), (11), the choices (6) for the elastic potential and (8) for the dissipation potential, and the constitutive assumption (19).

\subsection{Adhesion with purely normal damage and viscosity}

In the presence of viscous dissipation, the points of the $(\sigma, u)$ plane located above the loading curve become admissible. Then the restriction $u \leq \alpha$ is removed, and the state space becomes

$$
\Sigma=\left\{(u, \alpha) \quad \mid \quad u \geq 0, \quad 0 \leq \alpha \leq u_{r}\right\} .
$$

A consequence of this enlargement of the state space is that the state variable $\alpha$ is no longer identified with the largest displacement $u$ reached before the current instant.

For the strain energy we keep the expression (6), and for the dissipation potential due to damage we keep the expression (8). To it, we add the potential of the viscous dissipation

$$
\Phi_{\mathrm{v}}(\alpha, \dot{\alpha})=\frac{1}{4} h(\alpha) \dot{\alpha}^{2},
$$

with $h(\alpha)>0$. With this potential is associated the dissipation power

$$
D_{\mathrm{v}}(\alpha, \dot{\alpha})=\frac{\partial}{\partial \dot{\alpha}} \Phi_{\mathrm{v}}(\alpha, \dot{\alpha}) \dot{\alpha}=\frac{1}{2} h(\alpha) \dot{\alpha}^{2}
$$

The total dissipation power is then

$$
D=D_{\mathrm{d}}+D_{\mathrm{v}},
$$

with $D_{\mathrm{d}}$ as in (9). Notice that both contributions are governed by the same state variable.

The dissipation inequality $D_{\mathrm{d}} \geq 0$ still yields $\dot{\alpha} \geq 0$, and the power equation (16) now has an extra term due to viscous dissipation:

$$
(\sigma-g(\alpha) u) \dot{u}+\frac{1}{2} g^{\prime}(\alpha)\left(\alpha^{2}-u^{2}\right) \dot{\alpha}-\frac{1}{2} h(\alpha) \dot{\alpha}^{2}=0 \text {. }
$$

We keep the constitutive equation (19), which leads to the unilateral contact conditions (20). Then the power equation reduces to

$$
g^{\prime}(\alpha)\left(\alpha^{2}-u^{2}\right) \dot{\alpha}-h(\alpha) \dot{\alpha}^{2}=0 .
$$

Let us exclude the trivial solution of $\dot{\alpha}$ identically zero, which corresponds to a non-dissipative response. Then we determine separately the evolution of $\alpha$ in the three following subsets of the state space:

(i) In the subset $\left\{\alpha \leq u, 0 \leq \alpha<u_{r}\right\}$, the differential equation

$$
\dot{\alpha}=\frac{g^{\prime}(\alpha)}{h(\alpha)}\left(\alpha^{2}-u^{2}\right)
$$

holds. Indeed, the condition $\dot{\alpha} \geq 0$ is satisfied because $g^{\prime}(\alpha) / h(\alpha)<0$ and $\alpha^{2} \leq u^{2}$. Moreover, there is dissipation, $\dot{\alpha}>0$, only if $\alpha^{2}<u^{2}$. 
(ii) In the subset $\left\{u<\alpha, 0 \leq \alpha<u_{r}\right\}$, one has $h(\alpha) \dot{\alpha}^{2}=g^{\prime}(\alpha)\left(\alpha^{2}-u^{2}\right) \dot{\alpha} \leq 0$, with $h(\alpha)>0$. Then it must be $\dot{\alpha}=0$.

(iii) In the subset $\left\{\alpha=u_{r}\right\}$ one has $\dot{\alpha}=0$, because $\alpha$ cannot decrease by the dissipation inequality $\dot{\alpha} \geq 0$, and cannot increase because it cannot take values greater than $u_{r}$.

In conclusion, the evolution law is

$$
\dot{\alpha}=\left\{\begin{array}{cc}
-\frac{g^{\prime}(\alpha)}{h(\alpha)}\left(u^{2}-\alpha^{2}\right) & \text { if } \alpha<u, 0 \leq \alpha<u_{r}, \\
0 & \text { otherwise. }
\end{array}\right.
$$

This law has been obtained with the same assumptions made in the non-viscous case, except that the assumption $u \leq \alpha$ has been removed, a viscous dissipation potential has been added, and the trivial case of $\dot{\alpha}$ identically zero has been excluded.

The dotted line in Fig.2a is the response curve for the cyclic process shown in Fig. 2c, starting from the initial state $\left(u_{(0)}, \alpha_{0}\right)=\left(0, \alpha_{0}\right)$. In any response curve, the slope $g(\alpha)$ of the line joining the origin to the point $(\sigma, u)$ cannot increase in time. Indeed, its time derivative $g^{\prime}(\alpha) \dot{\alpha}$ is non-positive. Therefore, just like in the nonviscous case, the points located above the line $\sigma=g(\alpha) u$ are not accessible from the point $(\sigma, u)$.

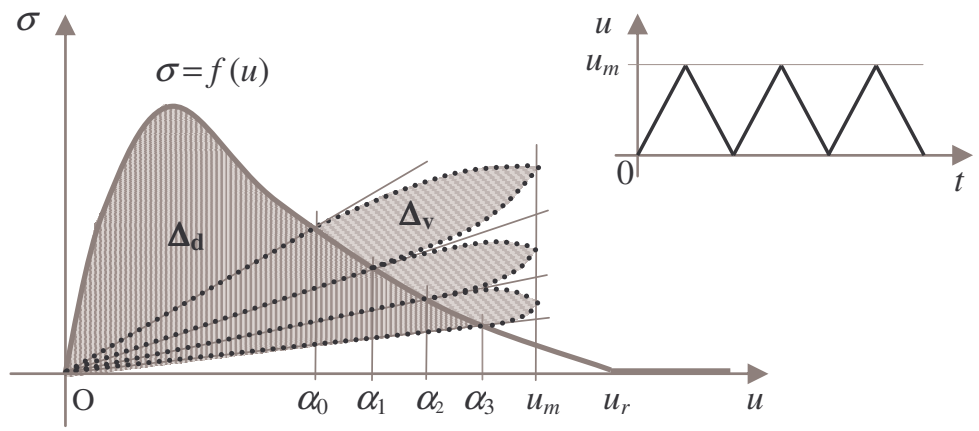

(a) (c)

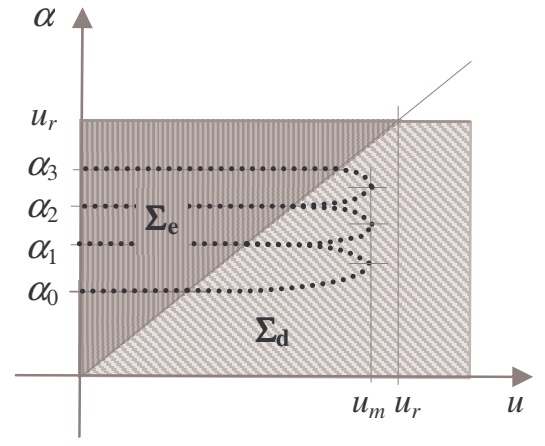

(b)

Figure 2. Adhesion with damage and viscosity. Response curve in the $(\sigma, u)$ plane, (a), and in the state space, (b), for the cyclic process shown in (c), under the initial condition $\alpha_{(0)}=\alpha_{0}$.

Consider a point $(\sigma, u)$ located above the loading curve $\sigma=f(u)$. For any such point, $u$ is greater than $\alpha$. Then for any response curve crossing this point, by differentiation of (18) one gets

$$
\dot{\sigma}=g(\alpha) \dot{u}+g^{\prime}(\alpha) u \dot{\alpha}=g(\alpha) \dot{u}-\frac{g^{\prime 2}(\alpha) u}{h(\alpha)}\left(u^{2}-\alpha^{2}\right) \leq g(\alpha) \dot{u} .
$$

This inequality shows that the slope $\dot{\sigma} / \dot{u}$ of the curve is never greater than the current elastic modulus $g(\alpha)$ at loading $(\dot{u}>0)$, and is always greater than the same modulus at unloading $(\dot{u} \leq 0)$. Consequently, every response curve located above the curve $\sigma=f(u)$ is star-shaped with respect to the origin. If, as in the case shown in the figure, there are several such curves, their union is star-shaped with respect to the origin as well.

The figure also shows the dissipation associated with the given process: the dissipation due to damage is given by the area $\Delta_{\mathrm{d}}$, and the dissipation due to viscosity is given by the area $\Delta_{\mathrm{V}}$. While $\Delta_{\mathrm{d}}$ is a function of the current state as in the non-viscous case, $\Delta_{\mathrm{v}}$ depends on the velocity $\dot{u}$.

The state space $\Sigma$ is the disjoint union of the two regions

$$
\Sigma_{\mathrm{e}}=\left\{(u, \alpha) \in \Sigma \mid u<\alpha, 0 \leq \alpha \leq u_{r}\right\}, \quad \Sigma_{\mathrm{d}}=\left\{(u, \alpha) \in \Sigma \mid \alpha \leq u, 0 \leq \alpha \leq u_{r}\right\} .
$$

As in the non-viscous case, $\Sigma_{\mathrm{e}}$ is the region of the non-dissipative processes, $\dot{\alpha}=0$. The dissipative processes, ( $\dot{\alpha}>0$ ), which in the non-viscous case were confined to the line $u=\alpha$, now spread over the whole region $\Sigma_{\mathrm{d}}$. The dotted curves in Fig. 2b are the counterparts of the response curves in Fig. 2a. By the evolution law (34), in the region $\Sigma_{\mathrm{d}}$ the slope $d \alpha / d u=\dot{\alpha} / \dot{u}$ increases with the distance $\left(u^{2}-\alpha^{2}\right)$ from the region $\Sigma_{\mathrm{e}}$, and is inversely proportional to the loading rate. In the limit case $\dot{u} \rightarrow+\infty$ the slope is zero; this means that there is no dissipation in infinitely fast processes. In the other limit case $\dot{u} \rightarrow 0$ the slope is $+\infty$. The corresponding slopes in the $(\sigma, u)$ plane can be deduced from equation (35). They are: $\dot{\sigma} / \dot{u}=g(\alpha)$ when $\dot{u} \rightarrow+\infty$, and $\dot{\sigma} / \dot{u}=-\infty$ when $\dot{u} \rightarrow 0$. The latter case describes the stress relaxation which occurs at fixed deformation. 


\subsection{Adhesion with normal and tangential damage}

In the presence of tangential forces $\tau$ and of tangential displacements $v$ we keep a single state variable $\alpha$, and we define a state of the interface to be the triplet $(u, v, \alpha)$. For $u$ we keep the non-interpenetration condition $u \geq 0$, and for $\alpha$ we keep the restrictions $0 \leq \alpha \leq \alpha_{r}$, where $\alpha_{r}$ is the critical value which determines the complete rupture of the interface.

The normal response $\sigma=f_{N}(u)$ and the tangential response $\tau=f_{T}(v)$ of the interface are described by two constitutive functions, $f_{N}$ and $f_{T}$. We assume that $f_{N}$ is defined over the non-negative $u$, and that it is positive in $\left(0, \alpha_{r}\right)$ and null outside. The function $f_{T}$ is defined over the whole real line and is odd, $f_{T}(-v)=-f_{T}(v)$, positive in $\left(0, \alpha_{r}\right)$, and null outside $\left(-\alpha_{r}, \alpha_{r}\right)$. We also assume that the restrictions of $f_{N}$ and $f_{T}$ to $\left(0, \alpha_{r}\right)$ are star-shaped with respect to the origin, so that the functions

$$
g_{N}(\alpha):=\frac{f_{N}(\alpha)}{\alpha}, \quad g_{T}(\alpha):=\frac{f_{T}(\alpha)}{\alpha},
$$

are strictly decreasing when $0 \leq \alpha<\alpha_{r}$.

The strain energy is taken to be the sum of a normal and a tangential part

$$
\Psi(u, v, \alpha)=\frac{1}{2} g_{N}(\alpha) u^{2}+\frac{1}{2} g_{T}(\alpha) v^{2},
$$

and the same holds for the dissipation potential

The latter corresponds to the dissipated work

$$
\Phi_{\mathrm{d}}(\alpha, \dot{\alpha})=-\frac{1}{2}\left(g^{\prime}{ }_{N}(\alpha)+g_{T}^{\prime}(\alpha)\right) \alpha^{2} \dot{\alpha} .
$$

$$
\Delta(\alpha)=\int_{0}^{\alpha}\left(f_{N}(s)+f_{T}(s)\right) d s-\frac{1}{2}\left(g_{N}(\alpha)+g_{T}(\alpha)\right) \alpha^{2} .
$$

and to the dissipation power

$$
D_{\mathrm{d}}(\alpha)=\dot{\Delta}(\alpha)=-\frac{1}{2}\left(g^{\prime}{ }_{N}(\alpha)+g_{T}^{\prime}(\alpha)\right) \alpha^{2} \dot{\alpha} .
$$

Note that since both $g_{N}$ and $g_{T}$ are strictly decreasing, the dissipation inequality $D_{\mathrm{d}}(\alpha) \geq 0$ still reduces to $\dot{\alpha} \geq 0$ when $\alpha>0$. The power equation now becomes

$$
\sigma \dot{u}+\tau \dot{v}=g_{N}(\alpha) u \dot{u}+g_{T}(\alpha) v \dot{v}+\frac{1}{2} g_{N}^{\prime}(\alpha)\left(u^{2}-\alpha^{2}\right) \dot{\alpha}+\frac{1}{2} g_{T}^{\prime}(\alpha)\left(v^{2}-\alpha^{2}\right) \dot{\alpha} .
$$

We take the constitutive laws

$$
\sigma^{+}=g_{N}(\alpha) u, \quad \tau=g_{T}(\alpha) v,
$$

keeping the possibility of a negative normal force $\sigma^{-}$when $u=\dot{u}=0$, see equations (20), (21). With these laws, equation (42) reduces to

and after setting

$$
\left(g_{N}{ }^{\prime}(\alpha)\left(u^{2}-\alpha^{2}\right)+g_{T}^{\prime}(\alpha)\left(v^{2}-\alpha^{2}\right)\right) \dot{\alpha}=0,
$$

$$
\rho(\alpha):=\frac{g^{\prime}{ }_{N}(\alpha)}{g^{\prime}{ }_{N}(\alpha)+g_{T}{ }_{T}(\alpha)}, \quad \varphi(u, v, \alpha):=\rho(\alpha) u^{2}+(1-\rho(\alpha)) v^{2}-\alpha^{2},
$$

the power equation takes the form

$$
\varphi(u, v, \alpha) \dot{\alpha}=0
$$

The state space is now

$$
\Sigma=\left\{(u, v, \alpha) \quad \mid \quad u \geq 0, \quad 0 \leq \alpha \leq \alpha_{r}, \quad \varphi(u, v, \alpha) \leq 0\right\} .
$$

This is a three-dimensional region, whose intersections with the planes $\alpha=$ const are half-ellipses. At the interior points of $\Sigma$ the strict inequality $\varphi(u, v, \alpha)<0$ holds, and equation (46) yields $\dot{\alpha}=0$. Therefore, there is no dissipation at the interior of $\Sigma$.

On the portion of the boundary at which $\varphi(u, v, \alpha)=0$, equation (46) is satisfied identically. To determine $\dot{\alpha}$ we follow the same procedure as in the purely normal case. From the vanishing of the second derivative of the energy, we obtain

$$
\left(\varphi_{u}(u, v, \alpha) \dot{u}+\varphi_{v}(u, v, \alpha) \dot{v}+\varphi_{\alpha}(u, v, \alpha) \dot{\alpha}\right) \dot{\alpha}=0,
$$

where the subscripts denote partial derivatives. We make the supplementary assumption

$$
\varphi_{c}(u, v, \alpha)<0 \quad \forall \alpha>0,
$$

under which the term between parentheses in (48) is strictly negative when $\varphi_{u}(u, v, \alpha) \dot{u}+\varphi_{v}(u, v, \alpha) \dot{v} \leq 0$. In this case the equality is satisfied only if $\dot{\alpha}=0$. If $\varphi_{u}(u, v, \alpha) \dot{u}+\varphi_{v}(u, v, \alpha) \dot{v}$ is positive, $\dot{\alpha}$ is determined by equating to 
zero the term between parentheses. Therefore, at the portion of the boundary at which $\varphi(u, v, \alpha)=0$ we have the evolution law

$$
\dot{\alpha}=\left\{\begin{array}{cc}
-\frac{\varphi_{u}(u, v, \alpha) \dot{u}+\varphi_{v}(u, v, \alpha) \dot{v}}{\varphi_{\alpha}(u, v, \alpha)} & \text { if } \varphi_{u}(u, v, \alpha) \dot{u}+\varphi_{v}(u, v, \alpha) \dot{v}>0, \\
0 & \text { otherwise. }
\end{array}\right.
$$

This law has the following geometrical interpretation: the vector $\left(\varphi_{u}, \varphi_{v}, \varphi_{\alpha}\right)$ is the exterior normal to the boundary, and its projection $N=\left(\varphi_{u}, \varphi_{v}, 0\right)$ on the plane $\alpha=$ const is the exterior normal to the intersection between the same plane and $\Sigma$. The assumption $\varphi_{\alpha}<0$ implies that the elliptic sections of $\Sigma$ expand with increasing $\alpha$, in such a way that the section at any $\alpha_{0}$ is strictly contained in the section at $\alpha$ for all $\alpha>\alpha_{0}$. In the language of plasticity, we are assuming an isotropic hardening law.

Let $\dot{U}=(\dot{u}, \dot{v})$ be a given increment of $U=(u, v)$. According to the law (50), if $\dot{U}$ points inwards $\Sigma$, $N(u, v, \alpha) \cdot \dot{U} \leq 0$, the evolution occurs at constant $\alpha$, that is, without dissipation. If $\dot{U}$ points outwards, $N(u, v, \alpha) \cdot \dot{U}>0$, the response is dissipative, with

$$
\dot{\alpha}=-\frac{N(u, v, \alpha) \cdot \dot{U}}{\varphi_{\alpha}(u, v, \alpha)} .
$$

Equation (48) tells us that in the dissipative case the vector $(\dot{u}, \dot{v}, \dot{\alpha})$ is orthogonal to the gradient of $\varphi$, and, therefore, tangent to the boundary. Thus, the dissipative response takes place on the boundary of $\Sigma$.

For $\alpha=0$, from (45) $)_{2}$ and (47) it follows that $u=v=0$, so that the cross-sectional ellipse reduces to a point. But at this point the gradient of $\varphi$ is zero, and equation (48) is satisfied identically. Then it becomes necessary to consider the third derivative of the energy equation (10), that is, the derivative of (48). It involves the second gradient of $\varphi$, which at $u=v=\alpha=0$ is

$$
\varphi_{u u}=2 \rho(0), \quad \varphi_{v v}=2(1-\rho(0)), \quad \varphi_{\alpha a}=-2, \quad \varphi_{u v}=\varphi_{u \alpha}=\varphi_{v \alpha}=0 .
$$

The corresponding evolution law is

$$
\dot{\alpha}^{2}=\rho(0) \dot{u}^{2}+(1-\rho(0)) \dot{v}^{2} .
$$

For $\alpha=\alpha_{r}$, we have $\dot{\alpha}=0$ as in the purely normal case. Therefore, the overall evolution law for the state variable $\alpha$ is

$$
\dot{\alpha}=\left\{\begin{array}{cc}
-\frac{N(u, v, \alpha) \cdot \dot{U}}{\varphi_{\alpha}(u, v, \alpha)} & \text { if } 0<\alpha<\alpha_{r}, \varphi(u, v, \alpha)=0, N(u, v, \alpha) \cdot \dot{U}>0, \\
\rho(0) \dot{u}^{2}+(1-\rho(0)) \dot{v}^{2} & \text { if } \alpha=0, \\
0 & \text { otherwise. }
\end{array}\right.
$$

Let us briefly discuss the physical meaning of the constitutive functions $f_{N}$ and $f_{T}$, and how to determine them experimentally. In the purely normal case, the equation of the loading curve was $\sigma=f(u)$, and $f$ was determined by a monotonic loading test. In the present case of coupled normal and tangential actions, one may consider a purely normal loading process with increasing $u$ and null $v$, as well as a purely tangential loading process with increasing $v$ and null $u$. Each process determines an evolution curve on the boundary region $\varphi(u, v, \alpha)=0$ of the state space $\Sigma$. For the first process, equation $(45)_{2}$ yields

$$
0=\varphi(u, 0, \alpha)=\rho(\alpha) u^{2}-\alpha^{2},
$$

which means that $u=\alpha \rho^{-1 / 2}(\alpha)$ instead of $u=\alpha$ as in the purely normal case. Therefore, the response curve $\sigma=g_{N}(\alpha) u$ does not coincide with the constitutive curve $\sigma=g_{N}(u) u=f_{N}(u)$. Similarly, the curve $\tau=g_{T}(\alpha) v$ does not coincide with the constitutive curve $\tau=g_{T}(v) v=f_{T}(v)$. However, taking an increasing "diagonal" loading with $u=v$, equation $(45)_{2}$ reduces to the equality

$$
0=\varphi(u, u, \alpha)=u^{2}-\alpha^{2},
$$

from which it follows that $u=v=\alpha$, and therefore $\sigma=g_{N}(u) u=f_{N}(u)$ and $\tau=g_{T}(v) v=f_{T}(v)$. Therefore, the constitutive functions $f_{N}$ and $f_{T}$ are determined by this special loading process. This means that, as anticipated in the Introduction, in the presence of both normal and tangential deformation the constitutive functions are not determined by two separate loading experiments, one for the normal and one for the tangential deformation, but by a single diagonal experiment. 


\subsection{An example of evolution under complex loading}

Consider an interface subject to normal and tangential damage, in the initial state $u=v=\alpha=0$. As an example, we determine the response of the interface to the process $t \mapsto\left(u_{(t)}, v_{(t)}\right)$ made of the following five steps:

$$
\begin{aligned}
& \{1\} u_{(t)} \text { from } 0 \text { to } u_{1}>0, \quad v_{(t)}=v_{1}=0, \\
& \text { \{2\} } u_{(t)}=u_{2}=u_{1}, \quad v_{(t)} \text { from } 0 \text { to } v_{2}>0 \text {, } \\
& \text { \{3\} } u_{(t)} \text { from } u_{2} \text { to } u_{3}>u_{2}, v_{(t)} \text { from } v_{2} \text { to } v_{3}=0 \text {, } \\
& \text { \{4\} } u_{(t)} \text { from } u_{3} \text { to } u_{4}=0, v_{(t)} \text { from } 0 \text { to } v_{4}<0 \text {, } \\
& \text { \{5\} } u_{(t)} \text { from } 0 \text { to } u_{5}=0, v_{(t)} \text { from } v_{4} \text { to } v_{5}<v_{4} \text {, }
\end{aligned}
$$

each of which performed at constant speed. For the sake of simplicity we assume that $\alpha_{r}=+\infty$, and we consider the special case of $g_{T}$ and $g_{N}$ proportional, $g_{T}(\alpha)=\lambda g_{N}(\alpha)$, in which $\rho(\alpha)$ takes the constant value $\rho=(1+\lambda)^{-1}$. In this case, the region $\Sigma$ is a cone with vertex at the origin. The cone is shown in Fig. 3a, where the process (57) is represented by the dotted line on the plane $(u, v)$. For constant $\rho$, the gradient of $\varphi$ reduces to

$$
\varphi_{u}=2 \rho u^{2}, \quad \varphi_{v}=2(1-\rho) v^{2}, \quad \varphi_{\alpha}=-2 \alpha,
$$

and the evolution law (50) for the boundary points takes the simplified form

$$
\alpha \dot{\alpha}=\rho u \dot{u}+(1-\rho) v \dot{v} \quad \text { if } \rho u \dot{u}+(1-\rho) v \dot{v}>0, \quad \dot{\alpha}=0 \text { otherwise. }
$$

If $\rho u_{(t)} \dot{u}+(1-\rho) v_{(t)} \dot{v}>0$ over the time interval $\left(t_{0}, t\right)$, by time integration we get

$$
\alpha_{(t)}=\left(\alpha_{(t))}^{2}+\rho\left(u_{(t)}^{2}-u_{(t 0)}^{2}\right)+(1-\rho)\left(v_{(t)}^{2}-v_{(t))}^{2}\right)\right)^{1 / 2} .
$$

The first step of the given process starts at the vertex of the cone with initial velocities $\dot{u}>0$ and $\dot{v}=0$. From equation (53) it follows that $\dot{\alpha}_{(0)}=\rho^{1 / 2} \dot{u}_{(0)}$, and a loading regime starts. At the subsequent instants, from (60) one has $\alpha_{(t)}=\rho^{1 / 2} u_{(t)}$, and at the end of the step one gets

$$
\alpha_{1}=\rho^{1 / 2} u_{1} .
$$

In the second step, since $\dot{u}=0$ and $v \dot{v}>0$, a new loading regime takes place. Again from (60) it turns out that, at the end of the step,

$$
\alpha_{2}=\left(\rho u_{2}^{2}+(1-\rho) v_{2}^{2}\right)^{1 / 2} \text {. }
$$

The third step has $\dot{u}>0$ and $v \dot{v}<0$. The assumption that the speed is constant throughout the step determines the proportionality relations

$$
\frac{\dot{v}}{\dot{u}}=\frac{v_{(t)}-v_{2}}{u_{(t)}-u_{2}}=\frac{-v_{2}}{u_{3}-u_{2}} .
$$
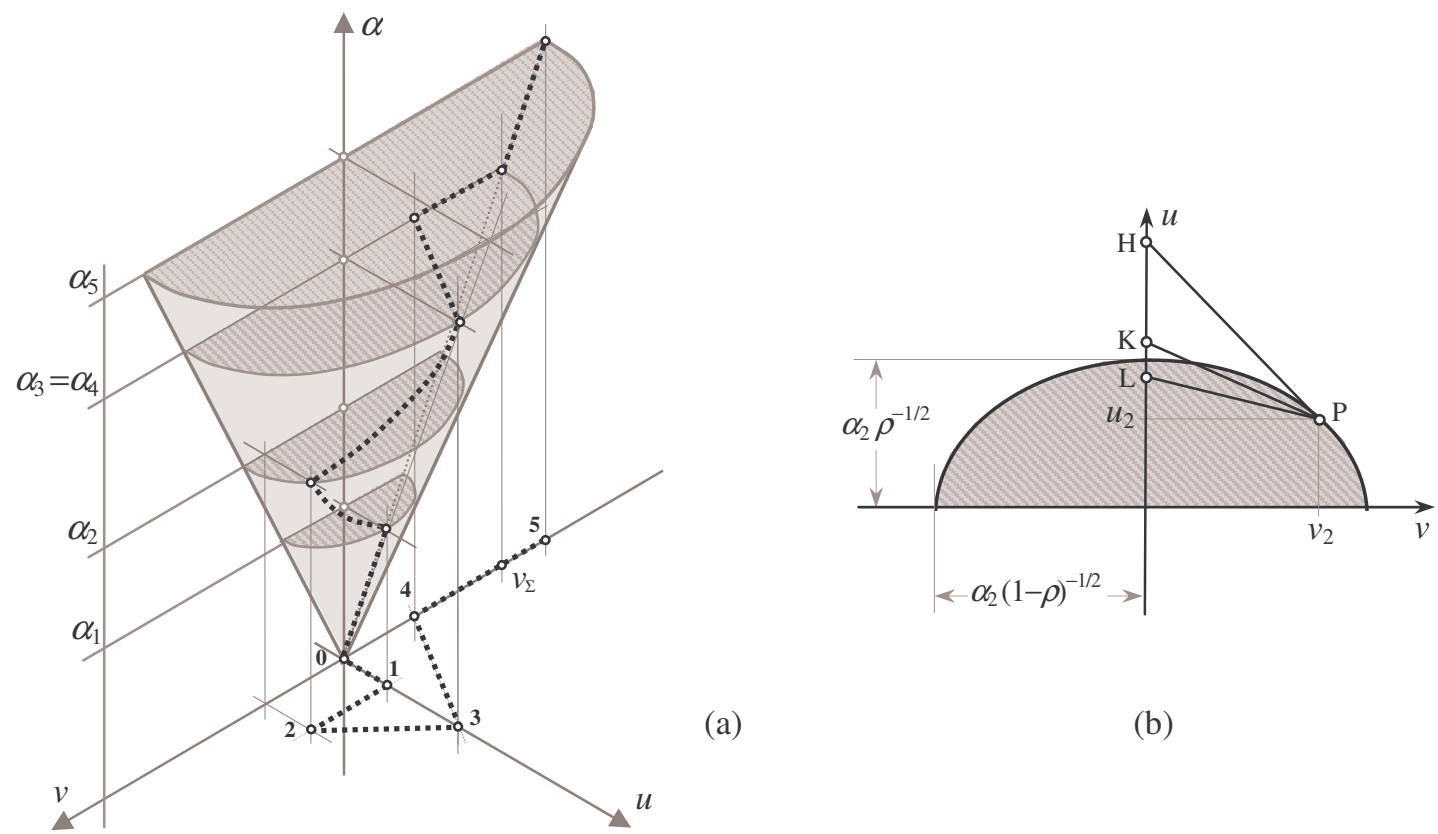

(a)

(b)

Figure 3. Evolution of $\alpha$ during the process (53) (a), and (b) the three possible patterns of evolution for the third step (in (a), the scale of $\alpha$ has been amplified) 
Then in equation (48) the term

has the same sign as the difference

$$
\varphi_{u} \dot{u}+\varphi_{v} \dot{v}=\rho u_{(t)} \dot{u}+(1-\rho) v_{(t)} \dot{v}
$$

$$
\rho u_{(t)}\left(u_{3}-u_{2}\right)-(1-\rho) v_{(t)} v_{2} .
$$

Therefore, it is the sign of this term which determines whether there is loading or unloading. In particular, at the initial instant of the third step there is loading if

$$
\rho u_{2}\left(u_{3}-u_{2}\right)-(1-\rho) v_{2}^{2} \geq 0
$$

and because the difference (65) increases with $t$, it remains positive during the whole step. This is the case represented by the line PH in Fig. 3b. On the contrary, if

$$
\rho u_{3}^{2} \leq \alpha_{2}^{2}=\rho u_{2}^{2}+(1-\rho) v_{2}^{2},
$$

then $\varphi\left(u_{3}, 0, \alpha_{2}\right)=\rho u_{3}^{2}-\alpha_{2}^{2}<0$, that is, the segment joining the points $\left(u_{2}, 0, \alpha_{2}\right),\left(u_{3}, 0, \alpha_{2}\right)$ belongs is interior to the state space $\Sigma$. In this case, the unloading regime represented by the line PL in the figure takes place. In the intermediate case

$$
\rho u_{2}\left(u_{3}-u_{2}\right)<(1-\rho) v_{2}^{2}<\rho\left(u_{3}^{2}-u_{2}^{2}\right),
$$

there is initial unloading, followed by a loading regime. The transition from unloading to loading occurs when the line PK intersects the ellipse. We consider the case (66) of loading over the whole step. At the end of the step, one has

$$
\alpha_{3}=\rho^{1 / 2} u_{3} .
$$

In the fourth step, one has $\dot{u}<0$ and $v \dot{v}>0$. Since the initial value of $v$ is zero, the term involving $\dot{u}$ is initially dominant. Therefore, an unloading regime takes place. If $\left|v_{4}\right|<\alpha_{3}(1-\rho)^{-1 / 2}$, this regime is maintained over the step, and the final value of $\alpha$ is $\alpha_{4}=\alpha_{3}$.

In the fifth and last step, one has $\dot{u}=0$ and $v \dot{v}>0$. Since the step starts at the interior of $\Sigma$, unloading will occur until the point $\left(0, v_{(t)}\right)$ reaches the boundary of $\Sigma$. This occurs when $v=v_{\Sigma}=-\alpha_{4}(1-\rho)^{-1 / 2}$. If $\left|v_{5}\right|>\left|v_{\Sigma}\right|$, a loading regime follows, at the end of which one has

$$
\alpha_{5}=-(1-\rho) v_{5}
$$
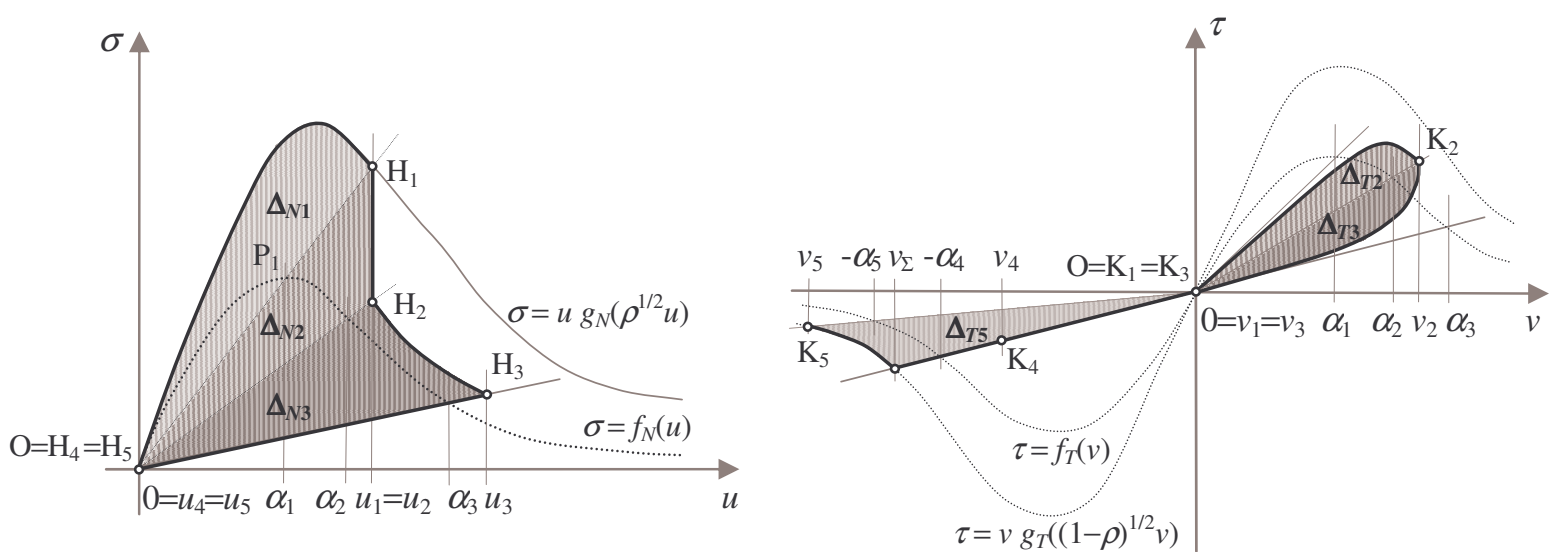

Figure 4. Normal and tangential response curves in the case of an interface subjected to the process (53) (the gray areas are the energies dissipated in the first three steps).

We now determine the response curves $(\sigma, u)$ and $(\tau, v)$. In the first step, since it was established that $\alpha_{(t)}=\rho^{1 / 2} u_{(t)}$, it follows from the constitutive equations (43) that

$$
\sigma=u g_{N}\left(\rho^{1 / 2} u\right), \quad \tau=0 .
$$

The first equation provides the expression for the loading curve for $u$ when $v=0$. This curve can be drawn up as shown in Fig. 4a. For a given $u_{1}$ and for $\alpha_{1}=\rho^{1 / 2} u_{1}$, the intersection $\mathrm{P}_{1}$ between the constitutive curve $\sigma=f_{N}(u)$ and the vertical from $\alpha_{1}$ determines the slope $g_{N}\left(\alpha_{1}\right)$, and the intersection $\mathrm{H}_{1}$ between the line $\mathrm{OP}_{1}$ with the vertical from $u_{l}$ determines a point on the loading curve. The entire loading curve can be drawn up by repeating this procedure for a sufficiently large number of values of $u$. 
In the first step, the loading curve $(71)_{1}$ is followed up to the point $\mathrm{H}_{1}$, while in the $(\tau, v)$ plane there is no evolution because $\tau_{(t)}=v_{(t)}=0$. In the second step, the response curve $\tau=g_{T}(\alpha) v$ starts at the origin with the slope $g_{T}\left(\alpha_{1}\right)$, and ends at the intersection $\mathrm{K}_{2}$ between the vertical from $v_{2}$ and the line from the origin with slope $g_{T}\left(\alpha_{2}\right)$, with $\alpha_{2}$ given by (57). In addition, since $u$ is constant, the response curve $\sigma=g_{N}(\alpha) u$ is the vertical segment starting from $\mathrm{H}_{1}$ and ending at the intersection $\mathrm{H}_{2}$ with the line from the origin with slope $g_{N}\left(\alpha_{2}\right)$.

In the third step, assuming that inequality (66) holds, there is a loading regime, in which the response curve for $\tau$ starts at $\mathrm{K}_{2}$ and ends at the origin, while the response curve for $\sigma$ starts at $\mathrm{H}_{2}$ and ends at the intersection $\mathrm{H}_{3}$ between the vertical from $u_{3}$ and the line from the origin with slope $g_{T}\left(\alpha_{3}\right)$, where $\alpha_{3}=\rho^{1 / 2} u_{3}$ is given by equation (69).

In the fourth step, there is an unloading regime. The two response curves lie on straight lines from the origin. More precisely, the curve $\sigma$ goes through the segment $\mathrm{H}_{3} \mathrm{O}$, and the curve $\tau$ starts from the origin with the slope $g_{T}\left(\alpha_{3}\right)$, which is the slope of the tangent to the curve at the end of the previous step. In the last step, the point $(\sigma, u)$ stays fixed at the origin, and the point $(\tau, v)$ goes through the same line as before up to $v=v_{\Sigma}$, and then continues along the loading curve $\tau=v g_{T}\left((1-\rho)^{1 / 2} v\right)$ up to the point $\mathrm{K}_{5}$.

In Fig. 4 it can be seen that the state variable $\alpha$ is given by the abscissas of the intersections of the curves $\sigma=f_{N}(u)$ and $\tau=f_{T}(u)$ with the lines connecting the origin to the points $(\sigma, u)$ and $(\tau, v)$, respectively. The areas below the response curves give the work performed. The dissipated energy is given by the gray areas: $\Delta_{N 1}$ in the first step, $\Delta_{N 2}+\Delta_{T 2}$ in the second, $\Delta_{N 3}+\Delta_{T 3}$ in the third, and $\Delta_{T 5}$ in the fifth step.

\subsection{Adhesion with damage, viscosity, and friction}

We now generalize the model with normal and tangential loading by introducing viscosity and friction. A state of the interface is still defined by the triplet $(u, v, \alpha)$. Viscosity is accounted for by adding the viscous dissipation power $D_{\mathrm{v}}$ given by (29). For friction, we add the frictional dissipation power

$$
D_{\mathrm{f}}\left(\alpha, \sigma^{-}, \dot{v}\right)=\mu(\alpha) \sigma^{-}|\dot{v}|,
$$

where $\sigma^{-}$is the contact pressure exerted on the interface, and $\mu$ is a function which measures the intensity of friction. We assume that $\mu$ is an increasing function, with $\mu(0)=0$ and $\mu\left(\alpha_{r}\right)=\mu_{\infty}$. Accordingly, friction gradually comes into the play when the adhesion decreases, and is fully operating only after the adhesion disappears. We recall that, because of conditions (16) and (18), the pressure $\sigma^{-}$may take arbitrary positive values if $u=\dot{u}=0$, and is zero otherwise. In the present model, when $u=\dot{u}=0$ the pressure $\sigma^{-}$is assumed to be a known applied load. Keeping the expression (38) for the strain energy and (41) for the dissipation power due to damage, the power equation becomes

$$
\sigma \dot{u}+\tau \dot{v}=g_{N}(\alpha) u \dot{u}+g_{T}(\alpha) v \dot{v}+\frac{1}{2} g_{N}^{\prime}(\alpha)\left(u^{2}-\alpha^{2}\right) \dot{\alpha}+\frac{1}{2} g_{T}^{\prime}(\alpha)\left(v^{2}-\alpha^{2}\right) \dot{\alpha}+\frac{1}{2} h(\alpha) \dot{\alpha}^{2}+\mu(\alpha) \sigma^{-}|\dot{v}| .
$$

For $\sigma$ we keep the constitutive law $\sigma^{+}=g_{N}(\alpha) u$, while $\tau$ is assumed to be the sum of two contributions, one due to damage and one due to friction:

$$
\tau=\tau_{\mathrm{d}}+\tau_{\mathrm{f}}, \quad \tau_{\mathrm{d}}=g_{T}(\alpha) v, \quad \tau_{\mathrm{f}}=\mu(\alpha) \sigma^{-} \operatorname{sgn} \dot{v} .
$$

With the constitutive assumptions just made, equation (73) reduces to

$$
\left(g_{N}{ }^{\prime}(\alpha)\left(u^{2}-\alpha^{2}\right)+g_{T}{ }^{\prime}(\alpha)\left(v^{2}-\alpha^{2}\right)\right) \dot{\alpha}+h(\alpha) \dot{\alpha}^{2}=0 .
$$

In addition, taking $\rho$ as in (45) and setting

$$
\kappa(\alpha):=-\frac{h(\alpha)}{g^{\prime}{ }_{N}(\alpha)+g_{T}^{\prime}(\alpha)},
$$

equation (73) further reduces to

$$
\left(\rho(\alpha) u^{2}+(1-\rho(\alpha)) v^{2}-\alpha^{2}\right) \dot{\alpha}-\kappa(\alpha) \dot{\alpha}^{2}=0 .
$$

It follows that $\dot{\alpha}=0$ if the term between parentheses is non-positive, and that

$$
\dot{\alpha}=\frac{1}{\kappa(\alpha)}\left(\rho(\alpha) u^{2}+(1-\rho(\alpha)) v^{2}-\alpha^{2}\right)
$$

if the same term is positive. The state space

is the disjoint union of the regions

$$
\Sigma=\left\{(u, v, \alpha) \quad \mid \quad u \geq 0,0 \leq \alpha \leq \alpha_{r}\right\}
$$




$$
\begin{gathered}
\Sigma_{\mathrm{e}}=\left\{(u, v, \alpha) \in \Sigma \quad \mid \quad \rho(\alpha) u^{2}+(1-\rho(\alpha)) v^{2} \leq \alpha^{2}, \quad \alpha \leq \alpha_{r}\right\}, \\
\Sigma_{\mathrm{d}}=\left\{(u, v, \alpha) \in \Sigma \quad \mid \quad \rho(\alpha) u^{2}+(1-\rho(\alpha)) v^{2}>\alpha^{2}, \alpha \leq \alpha_{r}\right\}, \\
\Sigma_{\mathrm{r}}=\left\{(u, v, \alpha) \in \Sigma \quad \mid \alpha=\alpha_{r}\right\} .
\end{gathered}
$$

In the first region we have $\dot{\alpha}=0$, while $\dot{\alpha}$ is positive and given by (78) in the second. Therefore, $\Sigma_{\mathrm{e}}$ is the region in which the non-dissipative processes occur, and $\Sigma_{\mathrm{d}}$ is the region where the dissipative processes occur. In the region $\Sigma_{\mathrm{r}}$ we have $g_{N}(\alpha)=g_{T}(\alpha)=0$ and, therefore, $\sigma=\tau=0$. This is the region of total rupture, at which $\dot{\alpha}=0$.

Moreover, when the conditions $u=\dot{u}=0$ for unilateral contact are satisfied, the evolution of $\alpha$ is governed by equation (78) with $u=0$, and the response $\tau$ is determined by equations (74), with $\sigma^{-}$a given function of time. Thus, the evolution law for $\dot{\alpha}$ is fully determined.

As an example, let us determine the evolution of $\alpha$ in the process (57) and from the initial state $(0,0,0)$, in the presence of viscosity and friction. The functions $\rho, \kappa$ and $\mu$ are assumed to be constants, and $\alpha_{r}$ is taken to be equal to $+\infty$. As shown in Subsection 2.4, $\rho=$ const implies that $g_{N}$ and $g_{T}$ are proportional. Moreover, by (76), $\kappa$ $=$ const implies that the viscosity $h$ is proportional to $g^{\prime}{ }_{N}+g^{\prime}{ }_{T}$.

Since the response is now rate-dependent, the initial time $t_{0}=0$ and the final instants $t_{1} \ldots t_{5}$ of all loading steps must be specified. If at each step the displacement rates $\dot{u}_{i}, \dot{v}_{i}$ are constant, one has

$$
u_{(t)}=u_{i-1}+\left(t-t_{i-1}\right) \dot{u}_{i}, \quad v_{(t)}=v_{i-1}+\left(t-t_{i-1}\right) \dot{v}_{i}, \quad \forall t \in\left(t_{i-1}, t_{i}\right),
$$

and the evolution law (72) gives

$$
\kappa \dot{\alpha}_{(t)}+\alpha_{(t)}^{2}=\rho u_{i-1}^{2}+(1-\rho) v_{i-1}^{2}+2\left(\rho u_{i-1} \dot{u}_{i}+(1-\rho) v_{i-1} \dot{v}_{i}\right)\left(t-t_{i-1}\right)+\left(\rho \dot{u}_{i}^{2}+(1-\rho) \dot{v}_{i}^{2}\right)\left(t-t_{i-1}\right)^{2} .
$$

By integrating this differential equation under the initial condition

$$
\alpha_{\left(t_{i-1}\right)}=\alpha_{i-1},
$$

the evolution of $\alpha$ during the $i^{\text {th }}$ time interval can be determined. For example, in the first step one has $t_{0}=u_{0}=v_{0}$ $=\alpha_{0}=0$ and $\dot{v}_{1}=0$. Then equation (82) reduces to the Riccati equation

$$
\kappa \dot{\alpha}_{(t)}+\alpha_{(t)}^{2}=\rho \dot{u}_{i}^{2} t^{2}
$$

subject to the initial condition $\alpha_{(0)}=0$. The solution of this problem determines the initial condition $\alpha_{1}$ for the next step. One may keep proceeding in this way as long as the trajectory $t \mapsto(u(t), v(t), \alpha(t))$ lies inside the region $\Sigma_{\mathrm{d}}$. This is the case in our example during the first three steps. In the fourth step, the direction of the process $t \mapsto(u(t), v(t))$ is such that the trajectory enters $\Sigma_{\mathrm{e}}$ at a time $t_{\Sigma}$. The process then continues with constant $\alpha$ up to the state $\left(0, v_{4}, \alpha_{4}\right)$, with $\alpha_{4}=\alpha_{\left(t_{\Sigma}\right)}$.

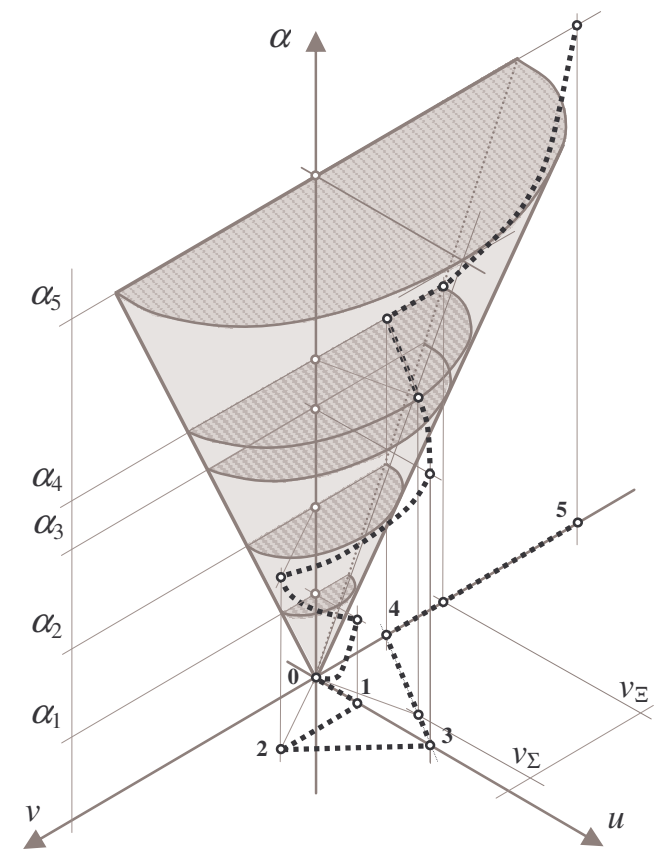

Figure 5. Evolution of $\alpha$ during the process (57), in the presence of viscosity and friction (the scale of $\alpha$ has been amplified). 
If $\alpha_{\Sigma}{ }^{2}<\rho u_{5}{ }^{2}$, there exists a time $t_{\Xi}$ during the fifth step, at which the trajectory leaves the region $\Sigma_{\mathrm{e}}$. At the instants $t>t_{\Xi}$, the values of $\alpha$ can be obtained by solving the differential problem

$$
\kappa \dot{\alpha}_{(t)}+\alpha_{(t)}^{2}=(1-\rho)\left(v_{\Xi}+\left(t-t_{\Xi}\right) \dot{v}_{4}\right)^{2}, \quad \alpha_{\left(t_{\Xi}\right)}=\alpha_{4} .
$$

The trajectory $t \mapsto\left(u_{(t)}, v_{(t)}, \alpha_{(t)}\right)$ is shown in Fig. 5 .

Let us now determine the response curves $(\sigma, u),\left(\tau_{\mathrm{d}}, v\right)$ and $\left(\tau_{\mathrm{f}}, v\right)$. In the first step of the process $(57)$, the response curve $(\sigma, u)$ starts from the origin and ends at the point $\mathrm{H}_{1}$ shown in Fig. 6.

The curve from $\mathrm{O}$ to $\mathrm{H}_{1}$ is obtained by evaluating $\alpha_{(t)}$ as the solution of equation (84) and substituting it into the constitutive law $\sigma_{(t)}=g_{N}\left(\alpha_{(t)}\right) u_{(t)}$. The trajectory goes beyond the elastic region $\Sigma_{\mathrm{e}}$, and the curve is located above the equilibrium curve $\sigma=u g_{N}\left(\rho^{1 / 2} u\right)$, at a distance proportional to the loading rate. In the figure, the final value $\alpha_{1}$ is given by the intersection between the line $\mathrm{OH}_{1}$ and the constitutive curve $\sigma=f_{N}(u)$. Throughout this step, the tangential displacement $v$ and the tangential force $\tau$ stay equal to zero.
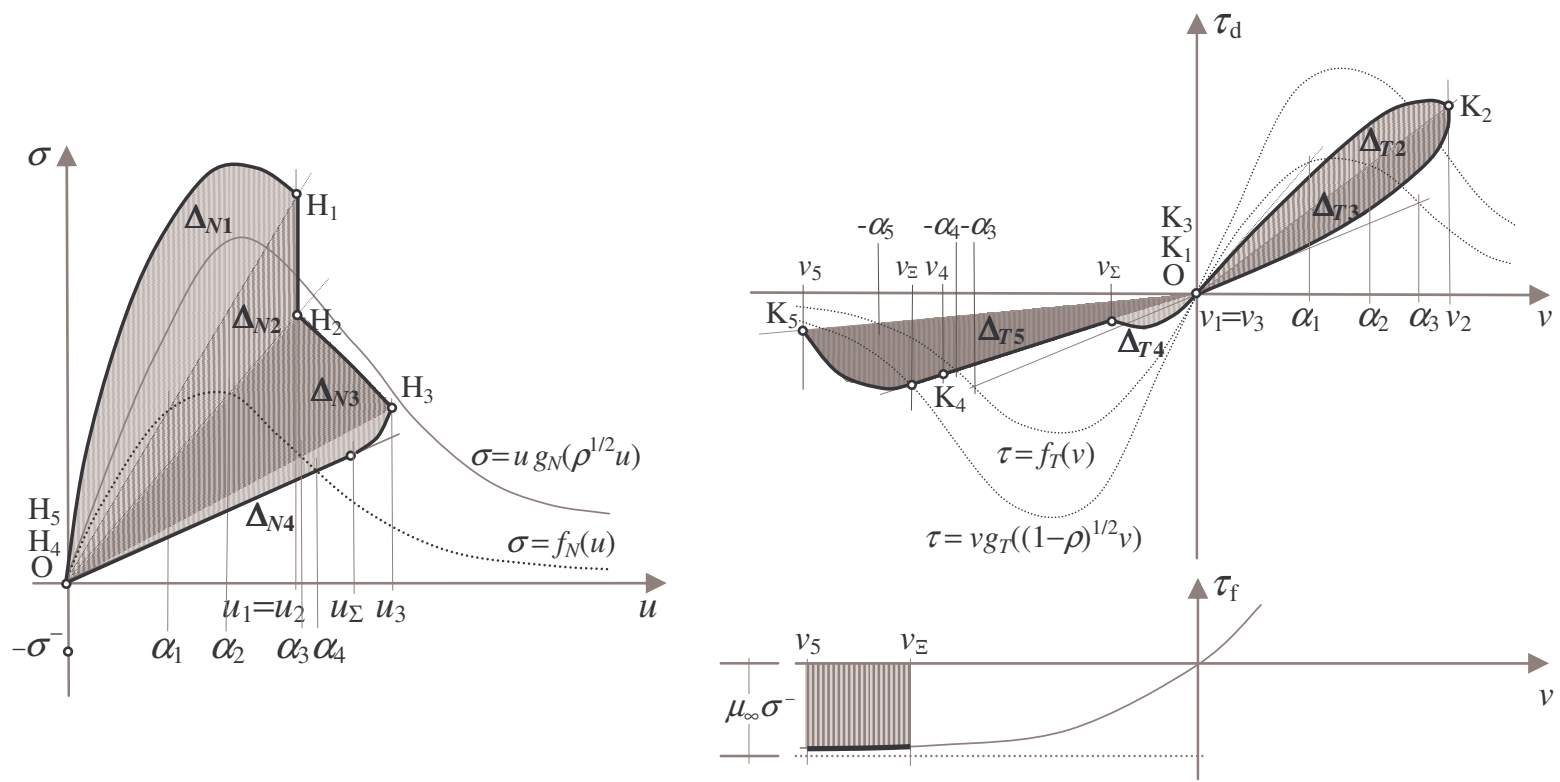

Figure 6. Curves of the normal and tangential responses for the process (57), in the presence of viscosity and friction.

In the second step, the evolution of $\tau_{\mathrm{d}}$ follows the curve $\tau_{\mathrm{d}(t)}=g_{T}\left(\alpha_{(t)}\right) v_{(t)}$, where $\alpha_{(t)}$ is the solution of problem (82), (83). The final value $\alpha_{2}$ is given by the intersection between the line $\mathrm{OK}_{2}$ and the loading curve $\tau_{\mathrm{d}}=f_{T}\left((1-\rho)^{1 / 2} v\right)$. The normal displacement $u$ remains constant, and $\sigma$ relaxes from $\mathrm{H}_{1}$ to the point $\mathrm{H}_{2}$ belonging to the half-line starting at the origin and crossing the curve $\sigma=f_{N}(u)$ at the point with abscissa $\alpha_{2}$.

In the third step we have the response curves $\mathrm{H}_{2} \mathrm{H}_{3}$ and $\mathrm{K}_{2} \mathrm{~K}_{3}$, with $\mathrm{K}_{3}$ coinciding with the origin because $v_{3}=0$. In the fourth step, at first there is a dissipative regime, which ends at the point $\left(u_{\Sigma}, v_{\Sigma}\right)$, and is followed by an elastic regime. The response curves are the segments $\mathrm{H}_{3} \mathrm{H}_{4}$ and $\mathrm{K}_{3} \mathrm{~K}_{4}$. Their slopes are determined by the value $\alpha_{4}$ of $\alpha$ given by the solution to the problem at the point $\left(u_{\Sigma}, v_{\Sigma}\right)$. In particular, $\mathrm{H}_{4}$ coincides with the origin.

Lastly, in the fifth step we have $u=\dot{u}=0$. There is no visco-elastic response, but the effects of unilateral contact are present. Therefore, there is a non-null $\tau_{\mathrm{f}}$. We assume that the force $\sigma^{-}$, which is an applied load, is constant in time. In the diagram $(\sigma, u)$, the visco-elastic response being zero, we only need to put the force $-\sigma^{-}$ on the vertical axis. In the diagram $\left(\tau_{\mathrm{f}}, v\right)$, we have the tangential force

given by the constitutive law $(74)_{3}$.

$$
\tau_{\mathrm{f}}=-\mu(\alpha) \sigma^{-}
$$

\section{The RCCM model.}

As a further example, we now discuss the RCCM model developed in (Raous et al 1999), (Raous and Monerie 2002), and we show that it is indeed a particular case of the general frame presented in this paper. In the RCCM model, the adhesion is characterized by a state variable $\beta$, representing the intensity of adhesion (Frémond 1987). The same state variable also controls viscosity and Coulomb friction. 
We show that the RCCM model can be deduced from the general model by making an appropriate choice of the strain energy, of the dissipation potentials, and of the constitutive functions $f_{N}$ and $f_{T}$. Let us first consider the basic model, without viscosity and friction. In it, a state of the interface is described by the triplet $(u, v, \beta)$. For the strain energy we take

$$
\Psi(u, v, \beta)=\frac{1}{2}\left(C_{N} u^{2}+C_{T} v^{2}\right) \beta^{2} .
$$

This is a special case of equation (38), with

$$
g_{N}(\alpha)=C_{N} \beta^{2}, \quad g_{T}(\alpha)=C_{T} \beta^{2} .
$$

Here $C_{N}$ and $C_{T}$ are given positive constants, representing the initial stiffnesses of the interface when the adhesion is complete. Taking $\beta$ to be a function of $\alpha$, by differentiation we get

$$
g^{\prime}{ }_{N}(\alpha)=2 C_{N} \beta(\alpha) \beta^{\prime}(\alpha), \quad g_{T}^{\prime}(\alpha)=2 C_{T} \beta(\alpha) \beta^{\prime}(\alpha) .
$$

The function $\rho(\alpha)$ introduced in (45) then takes the constant value

$$
\rho=\frac{C_{N}}{C_{N}+C_{T}} .
$$

For adhesive damage, in the RCCM model the dissipation potential

$$
\Phi_{\mathrm{d}}(\dot{\beta})=-\omega \dot{\beta}
$$

is taken, with $\omega$ a positive constant. This corresponds to Dupré's adhesive energy, in which an energy threshold for decohesion is introduced (Georges, 2000). From comparison with equation (40), we obtain

$$
\frac{1}{2}\left(g^{\prime}{ }_{N}(\alpha)+g_{T}^{\prime}(\alpha)\right) \alpha^{2} \dot{\alpha}=\omega \dot{\beta}=\omega \beta^{\prime}(\alpha) \dot{\alpha},
$$

and taking into account relations (89), the following relationship between the state variables $\alpha$ and $\beta$ follows

$$
\beta=\frac{\omega}{\left(C_{N}+C_{T}\right) \alpha^{2}}=: \frac{\omega^{*}}{\alpha^{2}} .
$$

The dissipation power due to damage is

$$
D_{\mathrm{d}}(\dot{\beta})=\frac{\partial}{\partial \dot{\beta}} \Phi_{\mathrm{d}}(\dot{\beta}) \dot{\beta}=-\omega \dot{\beta},
$$

and the dissipation inequality (11) gives $\dot{\beta} \leq 0$.

From (88) and (93) one can deduce the following dependence of $g_{N}$ and $g_{T}$ upon $\alpha$

$$
g_{N}(\alpha)=C_{N} \frac{\omega^{* 2}}{\alpha^{4}}, \quad g_{T}(\alpha)=C_{T} \frac{\omega^{* 2}}{\alpha^{4}},
$$

and the following forms of the constitutive functions $f_{N}$ and $f_{T}$ :

$$
f_{N}(u)=C_{N} \frac{\omega^{* 2}}{u^{3}}, \quad f_{T}(v)=C_{T} \frac{\omega^{* 2}}{v^{3}} .
$$

In the absence of viscosity and friction the state space is

where

$$
\Sigma=\{(u, v, \beta) \quad \mid \quad u \geq 0, \quad 0 \leq \beta \leq 1, \quad \psi(u, v, \beta) \leq 0\},
$$

$$
\psi(u, v, \beta)=C_{N} u^{2}+C_{T} v^{2}-\frac{\omega}{\beta},
$$

and the response of the interface is governed by the evolution law

$$
\dot{\beta}=\left\{\begin{array}{cc}
-2\left(C_{N} u \dot{u}+C_{T} v \dot{v}\right) \frac{\beta^{2}}{\omega} & \text { if } \psi(u, v, \beta)=0 \text { and } C_{N} u \dot{u}+C_{T} v \dot{v}>0, \\
0 & \text { otherwise. }
\end{array}\right.
$$

In the RCCM model, $\beta$ varies between 0 and 1 . Therefore, $\alpha$ varies from $\omega^{* 1 / 2}$ to $+\infty$. Thus, there is no complete rupture of the interface, since the critical value $\alpha=u_{r}$ is taken to be infinite. The restriction $\alpha \geq \omega^{* 1 / 2} \operatorname{does}$ not affect the substance of the model. Indeed, it simply corresponds to restricting the range of the possible initial values of $\alpha$. A consequence of this assumption is that the response to all processes $t \mapsto u_{(t)}$ developing inside the half-ellipse $C_{N} u^{2}+C_{T} v^{2} \leq \omega$ is linear elastic. The creation of such an elastic range is very convenient from the mechanical point of view. The modified normal and tangential constitutive curves are shown in Fig. 7.

Now consider the complete RCCM model, with viscosity and friction. The dissipation potential for the viscous dissipation is supposed to be

$$
\Phi_{\mathrm{v}}(\dot{\beta})=\frac{1}{2} b \dot{\beta}^{2},
$$



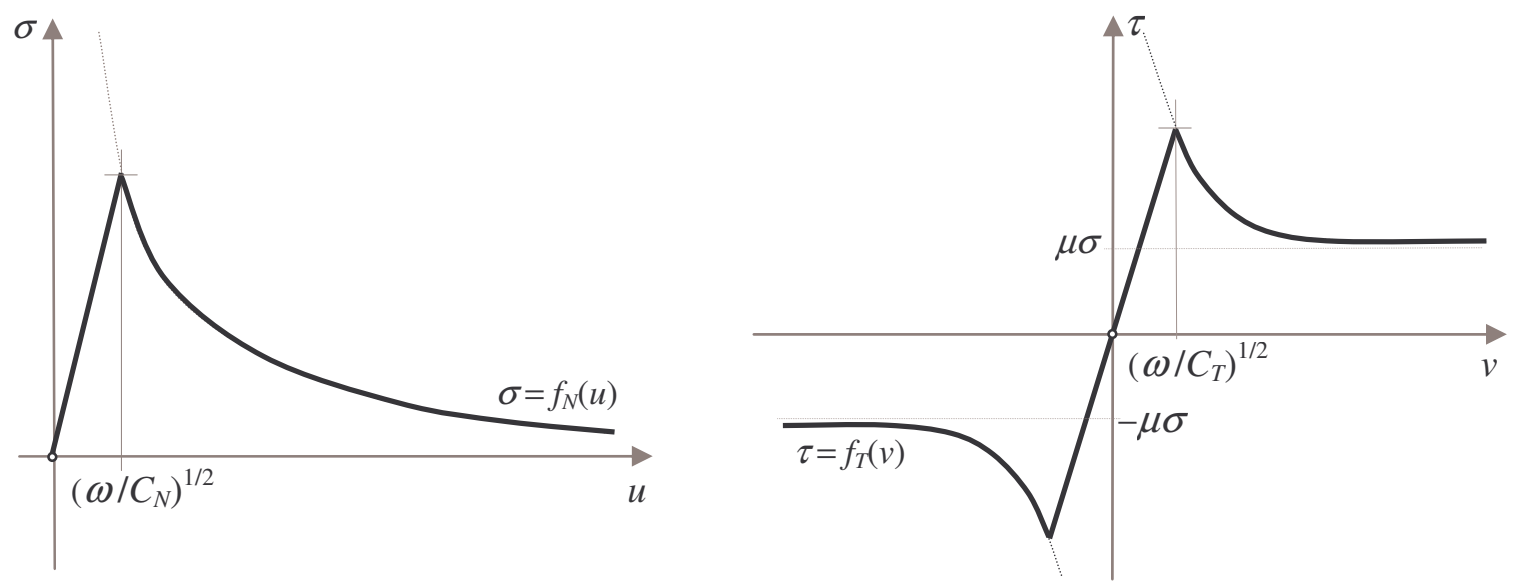

Figure 7. RCCM model. Modifications of the constitutive curves due to the assumption $\beta \leq 1$.

where $b$ is a positive constant, and the corresponding dissipation power is

$$
D_{\mathrm{v}}(\beta)=\dot{\beta} \frac{\partial}{\partial \dot{\beta}} \Phi_{\mathrm{v}}(\dot{\beta})=b \dot{\beta}^{2}
$$

By comparison with the potential (28), we obtain

$$
\frac{1}{4} h(\alpha) \dot{\alpha}^{2}=\frac{1}{2} b \dot{\beta}^{2}=\frac{1}{2} b \beta^{\prime}(\alpha)^{2} \dot{\alpha}^{2}=2 b \frac{\omega^{* 2}}{\alpha^{6}} \dot{\alpha}^{2}
$$

so that

$$
h(\alpha)=8 b \frac{\omega^{* 2}}{\alpha^{6}} .
$$

Again in the RCCM model, the dissipation potential due to friction is

$$
\Phi_{\mathrm{f}}(u, \beta, \dot{v}, \dot{\beta})=-(1-\beta) \mu\left(\sigma-C_{N} u \beta^{2}\right)|\dot{v}| \ln (|\dot{\beta}|),
$$

and to it corresponds the dissipation power

$$
D_{\mathrm{f}}(u, \beta, \dot{v})=\dot{\beta} \frac{\partial}{\partial \dot{\beta}} \Phi_{\mathrm{f}}(u, \beta, \dot{v}, \dot{\beta})=-(1-\beta) \mu\left(\sigma-C_{N} u \beta^{2}\right)|\dot{v}| .
$$

Here $\mu$ is a positive constant and (1- $\beta) \mu$ is the current value of the friction coefficient. The factor $(1-\beta)$ has the purpose of gradually increasing the intensity of friction when the adhesion decreases. Indeed, in this way there is no friction when adhesion is total, $\beta=1$, and the friction coefficient takes the largest value $\mu$ when there is no more adhesion, $\beta=0$.

From equations (93) and (95) it follows that $C_{N} u \beta^{2}=g_{N} u$. Then from the constitutive law $\sigma^{+}=g_{N} u$ it follows $\sigma-C_{N} u \beta^{2}=\sigma-\sigma^{+}=-\sigma^{-}$, and the expression for $D_{\mathrm{f}}$ reduces to

$$
D_{\mathrm{f}}\left(\beta, \sigma^{-}, \dot{v}\right)=(1-\beta) \mu \sigma^{-}|\dot{v}| \text {. }
$$

A comparison with the general expression (72) of the frictional dissipation power shows that $\mu(\alpha)$ is equal to (1- $\beta) \mu$. Therefore, by (93), in the RCCM model the function $\mu(\alpha)$ has the form

$$
\mu(\alpha)=\left(1-\omega^{*} \alpha^{-2}\right) \mu .
$$

The evolution law for the state variable requires that there is no dissipation if $\psi(u, v, \beta)<0$ and that there is dissipation if $\psi(u, v, \beta)>0$. In the dissipative case, from (93), (98), and from the relation (78) of the general model it follows that

$$
\dot{\alpha}=\frac{1}{\kappa(\alpha)}\left(\frac{C_{N} u^{2}+C_{T} v^{2}}{C_{N}+C_{T}}-\alpha^{2}\right)=\frac{C_{N} u^{2}+C_{T} v^{2}-\omega / \beta}{\kappa(\alpha)\left(C_{N}+C_{T}\right)},
$$

and, from (76) and (93),

$$
\dot{\beta}=\beta^{\prime}(\alpha) \dot{\alpha}=-2 \frac{\omega^{*}}{\alpha^{3}} \dot{\alpha}, \quad \kappa(\alpha)=\frac{2 b}{\left(C_{N}+C_{T}\right) \alpha}
$$

Then the evolution law is 


$$
\dot{\beta}=\left\{\begin{array}{cc}
-\frac{\beta}{b}\left(C_{N} u^{2}+C_{T} v^{2}-\frac{\omega}{\beta}\right) & \text { if } \psi(u, v, \beta)>0, \\
0 & \text { otherwise. }
\end{array}\right.
$$

This law, which here has been deduced from the general model, is precisely the law assumed in (Raous et al 1999). Thus, we may conclude that the RCCM model is indeed a special case of the general scheme.

It should be noted that in (Raous et al 1999) and (Raous et Monerie 2002), as well as in (Frémond 1987), the strain energy was written in the form

$$
\Psi(u, v, \beta)=\frac{1}{2}\left(C_{N} \beta^{2} u^{2}+C_{T} \beta^{2} v^{2}\right)+\omega(1-\beta),
$$

while in the new formulation the second term is the dissipation due to damage. The new formulation is more natural, because condition $\dot{\beta} \leq 0$ is obtained directly from (12) and (93), whereas in (Raous et al 1999) the same condition was imposed by introducing a supplementary term in the dissipation potential. Moreover, the energetic term associated with adhesive damage is now conveniently included in the dissipation potential $\Phi$ and not in the free energy $\Psi$, as it was in the original formulation of the RCCM model.

To conclude, in Fig. 8 we give the graph of the evolution of $\beta$ in the process (57). This is simply the graph in Fig. 5 modified according to the change of variable (93). It is worth noting that, to be consistent with the restriction $\beta \leq 1$ present in the RCCM model, one is obliged to start from an initial value of $\alpha$ which is greater or equal to $\omega^{*}{ }^{1 / 2}$. In the present example the initial value $\alpha_{0}=\omega^{*}{ }^{1 / 2}$, corresponding to $\beta=1$, is taken. Moreover, the normal displacement $u_{1}$ is taken to be equal to $\left(\omega / C_{N}\right)^{1 / 2}$, so that the first step ends at the point $\left(u_{1}, v_{1}, \beta_{1}\right)=$ $\left(\left(\omega / C_{N}\right)^{1 / 2}, 0,1\right)$ located on the boundary of $\Sigma_{\mathrm{e}}$ and shown in Fig.7.

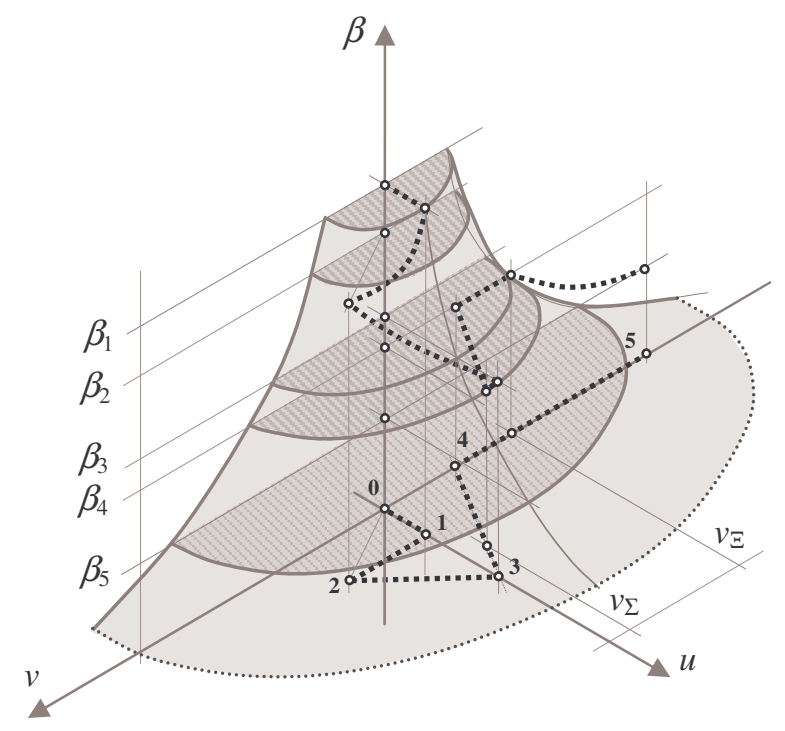

Figure 8. RCCM model. Evolution of $\beta$ during the process (57) in the presence of viscosity and friction.

\section{Conclusions}

A relatively simple and quite general form for models describing the response of an adhesive interface has been constructed. It is mainly based on given functions $f$ and $g$ which can be obtained through experiments related to normal and tangential loadings applied on the interface. The adhesive dissipation is directly connected to these functions. Viscosity and friction effects have been added through convenient choices for viscous and frictional dissipation potentials.

The general frame developed here provides a highly flexible tool for describing a wide range of experimentally observed interface behavior. Besides damage, viscosity and friction, other effects can be taken into account by introducing appropriate potentials and more sophisticated responses can be obtained by introducing supplementary state variables. Generalization to 3D models and to deformable bodies can be made in the same way as for the RCCM model in (Raous, 1999; Raous et al, 1999; Raous and Monerie, 2002). 


\section{References}

Allix, O., Ladevèze, P., Corigliano, A., 1995. Damage analysis of interlaminar fracture specimens. Composite Structures 31(1), pp. 61-74.

Barenblatt, G.I., 1962. The mathematical theory of equilibrium cracks in brittle fracture. Adv. Appl. Mechanics 7, pp. 55-129.

Brinckmann, S., Siegmund Th., 2008. A cohesive zone model based on the micromechanics of dislocations. Modelling Simul. Mater. Sci. Eng. 16, 065003, 19p.

Campillo, M., Ionescu, I. R., 1997. Initiation of antiplane shear instability under slip dependent friction. J. Geophys. Res. 102(B9), pp. 20363-20371.

Chaboche, J.-L., Feyel, F., Monerie, Y., 2001. Interface debonding model: a viscous regularization with a limited rate dependency. Int. J. Solids Structures 38(18), pp. 3127-3160.

Costanzo, F., Walton, J.R., 1997. A study of dynamic crack growth in elastic materials using a cohesive zone model. Int. J. Engng. Sci. 35(12-13), pp. 1085-1114.

Dugdale, D.S., 1960. Yielding of steel sheets containing slits. J. Mech. Phys. Solids 8, pp. 100-104.

Freddi, F., Frémond, M., 2006. Damage in domains and interfaces : a coupled predictive theory. J. Mech. Mat. Structures 1, pp. 1205-1234.

Frémond, M., 1987. Adhérence des solides. J. Méc. Théor. Appl. 6 (3), pp. 383-407.

Frémond, M., 1988. Contact with adhesion, in: Moreau, J.-J., Panagiotopoulos, P.D. (Eds.), Nonsmooth Mechanics and Applications, CISM Courses and Lectures, 302, Springer, Wien, pp. 177-221.

Georges, J.-M., 2000. Frottement, usure et lubrification. CNRS-Editions \& Eyrolles, Paris.

Halphen, B., Nguyen, Q.S., 1975. Sur les matériaux standard généralisés. J. de Mécanique 14, pp. 39-63.

Michel, J.C., Suquet, P., 1994. Analytical and numerical study of the overall behaviour of metal-matrix composites. Modelling. Simul. Mater. Sci. Eng. 2, pp. 637-658.

Monerie, Y., 2000. Fissuration des matériaux composites: rôle de l'interface fibre/matrice. Thesis, Université Aix-Marseille I.

Needleman, A., 1987. A continuum model for void nucleation by inclusion debonding. J. Appl. Mech. 54, pp. 525-531.

Needleman, A., 1990. An analysis of tensile decohesion along an interface. J. Mech. Phys. Solids 38(3), pp. 289-324.

Needleman, A., 1992. Micromechanical modeling of interfacial decohesion. Ultramicroscopy, 40, pp. $203-214$.

Needleman, A., Rosakis, A.J., 1999. The effect of bond strength and loading rate on the conditions governing the attainment of intersonic crack growth along interfaces. J. Mech. Phys. Solids 47, pp. 2411-2449.

Nguyen, C., Levy, A.J., 2009. An exact theory of interfacial debonding in layered elastic composites. Int. J. Solids Structures 46, pp. 2712-2723.

Péralès, F., 2005. Fissuration des matériaux à gradient de propriétés. Application au Zircaloy hydruré. Thesis, Université Montpellier II.

Raous, M., 1999. Quasistatic Signorini problem with Coulomb friction and coupling to adhesion, in: New Developments in Contact Problems, Wriggers, P., Panagiotopoulos, P.P. (Eds), CISM Courses and Lectures, 384, Springer Verlag, Wien, pp. 101-178.

Raous, M., Cangémi L., Cocou M., 1999. A consistent model coupling adhesion, friction and unilateral contact. Comput. Methods in Appl. Mech. Engrg. 177, pp 383-399.

Raous, M., Monerie, Y., 2002. Unilateral contact, friction and adhesion in composite materials, in: Martins, J.A.C., Monteiro Marques, M. (Eds), Contact Mechanics, Kluwer, Dordrecht, pp. 333-346.

Raous, M., Schryve, M., Cocou, M., 2006. Restorable adhesion and friction, in: Baniotopoulos, C.C. (Ed.), Nonsmooth/Nonconvex Mechanics with Applications in Engineering. Ziti Publisher, Thessaloniki, pp. 165172. 
Rice, J.R., Ruina A.L., 1983. Stability od steady frictional slipping. J. Appl. Mech. 50, pp. 343-349.

Talon, C., Curnier, A., 2003. A model of adhesion coupled to contact and friction. Eur. J. Mechanics A/Solids 22, pp. 545-565.

Truesdell, C., Noll, W., 1965. The non-linear field theories of mechanics, in: Handbuch der Physik, III/3, Springer, Berlin

Tvergaard, V., 1990. Effect of fibre debonding in a whisker-reinforced metal. Mat. Sci. Engrg. A125, pp. 203213.

Tvergaard, V., Hutchinson, J.W., 1992. The relation between crack growth resistance and fracture process parameters in elastic-plastic solids. J. Mech. Phys. Solids 40, pp.1377-1397.

Tvergaard, V., Hutchinson, J.W., 1996. Effects of strain-dependent cohesive zone model on prediction of crack growth resistance. Int. J. Solids Structures 33, pp. 3297-3308.

Uenishi, K., Rice J.R., 2003. Universal nucleation length for slip-weakening rupture instability under non uniform fault loading. J. Geophys. Res. 108(B1), pp. 2042, 2003.

Xu, X.-P., Needleman, A., 1994. Numerical simulations of fast crack growth in brittle solids. J. Mech. Phys. Solids 42(9), pp. 1397-1434. 